SLAC-PUB-7379, WIS-96/49/Dec-PH, CERN-TH/96-368, hep-ph/9701231

\title{
CP Violation Beyond the Standard Model
}

\author{
Yuval Grossman ${ }^{a}$, Yosef $\operatorname{Nir}^{b}$ and Riccardo Rattazzi ${ }^{c}$ \\ ${ }^{a}$ Stanford Linear Accelerator Center, Stanford University, Stanford, CA 94309, USA \\ ${ }^{b}$ Department of Particle Physics, Weizmann Institute of Science, Rehovot 76100, Israel \\ ${ }^{c}$ Theory Division, CERN, CH-1211 Geneva 23, Switzerland
}

\begin{abstract}
We review CP violation in various extensions of the electroweak sector of the Standard Model. A particular emphasis is put on supersymmetric models. We describe the two CP problems of supersymmetry, concerning $d_{N}$ and $\varepsilon_{K}$. We critically review the various mechanisms that have been suggested to solve these problems: exact universality, approximate CP symmetry, alignment, approximate universality and heavy squarks. We explain how future measurements of $\mathrm{CP}$ violation will test these mechanisms. We describe extensions of the quark sector and their implications on CP asymmetries in neutral $B$ decays, on the $K_{L} \rightarrow \pi \nu \bar{\nu}$ decay and on $\Delta \Gamma\left(B_{s}\right)$. We discuss CP violation in charged scalar exchange in models with natural flavor conservation and explain how transverse lepton polarization in meson decays can probe such models. CP violation in neutral scalar exchange arises in models of horizontal symmetries and may be manifest in heavy quark $(b$ and $t)$ physics. We describe the implications of Left-Right Symmetric models on $d_{N}, \varepsilon_{K}, \varepsilon^{\prime} / \varepsilon$ and CP asymmetries in $B$ decays. Finally, we briefly discuss the potential of future measurements of CP violation to discover New Physics.
\end{abstract}

To appear in the Review Volume "Heavy Flavours II", eds. A.J. Buras and M. Lindner, Advanced Series on Directions in High Energy Physics, World Scientific Publishing Co., Singapore. 


\section{Introduction}

$\mathrm{CP}$ violation is one of the most promising directions in the search for New Physics beyond the Standard Model.

- Experimentally, the Standard Model (SM) picture of CP violation has not been tested yet. At present, there is only a single (complex) $\mathrm{CP}$ violating parameter that has been measured [1]. This is $\varepsilon_{K}$ of the neutral $K$ system. Within the Standard Model, the existing measurements merely fix the value of the $\mathrm{CP}$ violating phase $\delta_{\mathrm{KM}}$ in the CabibboKobayashi-Maskawa (CKM) mixing matrix for quarks [2] but cannot test the prediction that $\delta_{\mathrm{KM}}$ constitutes the only source of $\mathrm{CP}$ violation.1. 1 A genuine testing of the KM picture of $\mathrm{CP}$ violation awaits the building of $B$ factories that would provide a second, independent measurement of $\mathrm{CP}$ violation [3].

- The observed baryon asymmetry of the Universe, if dynamically generated, requires that $\mathrm{CP}$ is violated [4]. The Standard Model CP violation, closely related to highly suppressed flavor changing processes, fails to produce this asymmetry by many orders of magnitude. (For recent discussions, see [5-7].) In contrast, various extensions of the SM and, in particular, models of New Physics close to the electroweak scale, provide new sources of $\mathrm{CP}$ violation that are large enough to be consistent with the observed asymmetry (for a recent review, see [8]). In some models, phases that are large enough to generate the baryon asymmetry also induce an electric dipole moment (EDM) of the neutron not far below the present experimental bound [9, 10].

- The QCD lagrangian does allow an additional source of CP violation, that is the $\theta_{\mathrm{QCD}}$ parameter. However, an extreme fine-tuning is needed in order that its contribution to the electric dipole moment of the neutron $d_{N}$ does not exceed the experimental upper bound. Various mechanisms that go beyond the SM, e.g. a Peccei-Quinn symmetry, spontaneous CP violation, or a vanishing mass of the up quark, may solve the problem.

- Almost any extension of the SM has, in general, new CP violating phases.

In this review we describe various extensions of the Standard Model and their implications on $\mathrm{CP}$ violation. We do not discuss in any detail either baryogenesis or the strong $\mathrm{CP}$

1 A large value of $\varepsilon_{K}$ would be inconsistent with the SM. However, any value $\left|\varepsilon_{K}\right| \leq \mathcal{O}\left(10^{-3}\right)$ can be accommodated. 
problem. Instead, we focus on extensions of the electroweak sector and their implications for the EDM of the neutron which, if measured in the foreseeable future, will clearly signal New Physics, and for CP violation in neutral meson mixing which, in some cases, is free from hadronic uncertainties and therefore could distinguish between Standard Model and New Physics contributions.

In section 2 we give a detailed and critical discussion of $\mathrm{CP}$ violation (and flavor problems) in Supersymmetry. We describe the supersymmetric CP problem, that is the generically too large supersymmetric contributions to the electric dipole moment of the neutron, and the supersymmetric $\varepsilon_{K}$ problem. We present five classes of supersymmetric models that solve or relax these problems: exact universality, approximate CP symmetry, alignment, approximate universality and heavy squarks. We explain how future measurements of $\mathrm{CP}$ violation will test these models.

Section 3 is devoted to extensions of the fermion sector. In particular, we consider additional $S U(2)$-singlet down quarks. Our emphasis here is on CP asymmetries in neutral $B$ decays into final CP eigenstates. These can be dramatically modified in such extensions. For certain decay modes, the measurement of the asymmetries can cleanly determine the relevant parameters of the extended sector. We also discuss the decay $K_{L} \rightarrow \pi \nu \bar{\nu}$ and $\mathrm{CP}$ violation in the width difference $\Delta \Gamma\left(B_{s}\right)$.

In section 4 we discuss extensions of the scalar sector. A model of spontaneous CP violation and Natural Flavor Conservation (NFC), where CP violation arises from charged scalar exchange only, provides us with an example of how CP violation can actually rule out various extensions of the Standard Model. We also explain how CP violation in charged scalar exchange may affect transverse lepton polarization. Then we discuss CP violation in neutral scalar exchange in models where approximate flavor symmetries (invoked to explain the smallness of the quark and lepton masses and mixing) replace NFC in suppressing flavor changing neutral current (FCNC) processes. We finally describe the idea of superweak $\mathrm{CP}$ violation with emphasis on the fact that it refers to many different types of models.

In section 5 we briefly discuss an extension of the gauge sector. We describe a LeftRight Symmetric model (LRS) where CP is spontaneously broken. We argue that, when $\mathrm{CP}$ violation arises in non-horizontal gauge interactions, the effects in the $B$ system are 
likely to be small.

Our conclusions are given in section 6 , where we present the various future measurements of CP violation with emphasis on their potential to discover effects of New Physics.

\section{Supersymmetry}

A generic supersymmetric extension of the Standard Model contains a host of new flavor and CP violating parameters (for reviews on supersymmetry see refs. [11-14]). The requirement of consistency with experimental data provides strong constraints on many of these parameters. For this reason, the physics of flavor and CP violation has had a profound impact on supersymmetric model building. A discussion of $\mathrm{CP}$ violation in this context can hardly avoid addressing the flavor problem itself. Indeed, many of the supersymmetric models that we analyze below were originally aimed at solving flavor problems.

As concerns CP violation, one can distinguish two classes of experimental constraints. First, bounds on nuclear and atomic electric dipole moments determine what is usually called the supersymmetric CP problem. Second, the physics of neutral mesons and, most importantly, the small experimental value of $\varepsilon_{K}$ pose the supersymmetric $\varepsilon_{K}$ problem. In the next two subsections we describe the two problems.

In most of the literature, solutions to these two problems are discussed separately. We believe, however, that since they represent the same issue, i.e. the origin of $\mathrm{CP}$ violation, and since, in general, the mechanisms that solve them can be classified in similar ways, they should be discussed together to get the appropriate picture of SUSY CP violation. Thus, we analyze in turn five classes of supersymmetric models and all aspects of CP violation for each of them.

Before turning to a detailed discussion, we define two scales that play an important

role in supersymmetry: $\Lambda_{S}$, where the soft supersymmetry breaking terms are generated, and $\Lambda_{F}$, where flavor dynamics takes place. When $\Lambda_{F} \gg \Lambda_{S}$, it is possible that there are no genuinely new sources of flavor and CP violation. This leads to models with exact universality, which we discuss in section 2.3. When $\Lambda_{F} \lesssim \Lambda_{S}$, we do not expect, in general, that flavor and $\mathrm{CP}$ violation are limited to the Yukawa matrices. One way to suppress 
$\mathrm{CP}$ violation would be to assume that $\mathrm{CP}$ is an approximate symmetry of the full theory (namely, CP violating phases are all small). We discuss this scenario in section 2.4. Another option is to assume that, similarly to the Standard Model, CP violating phases are large, but their effects are screened, possibly by the same physics that explains the various flavor puzzles. Such models, with Abelian or non-Abelian horizontal symmetries, are described in sections 2.5 and 2.6, respectively. Finally, it is possible that $\mathrm{CP}$ violating effects are suppressed because squarks are heavy. This scenario is discussed in section 2.7.

\subsection{The Supersymmetric CP Problem}

One aspect of supersymmetric CP violation involves effects that are flavor preserving. Then, for simplicity, we describe this aspect in a supersymmetric model without additional flavor mixings, i.e. the minimal supersymmetric standard model (MSSM) with universal sfermion masses and with the trilinear SUSY-breaking scalar couplings proportional to the corresponding Yukawa couplings. (The generalization to the case of non-universal soft terms is straightforward.) In such a constrained framework, there are four new phases beyond the two phases of the Standard Model $\left(\delta_{\mathrm{KM}}\right.$ and $\left.\theta_{\mathrm{QCD}}\right)$. One arises in the bilinear $\mu$-term of the superpotential,

$$
W=\mu H_{u} H_{d}
$$

while the other three arise in the soft supersymmetry breaking parameters $m_{\tilde{g}}$ (the gaugino mass), $A$ (the trilinear scalar coupling) and $m_{12}^{2}$ (the bilinear scalar coupling):

$$
\mathcal{L}=-\frac{1}{2} m_{\tilde{g}} \tilde{g} \tilde{g}-A\left(Y^{u} Q H_{u} \bar{u}-Y^{d} Q H_{d} \bar{d}-Y^{e} L H_{d} \bar{\ell}\right)-m_{12}^{2} H_{u} H_{d}+\text { h.c. },
$$

where $\tilde{g}$ are the gauginos and $Y$ are Yukawa matrices. Only two combinations of the four phases are physical [15]. This can be easily shown by following the discussion of ref. [16]. In the absence of (2.1) and (2.2), there are two additional global $U(1)$ symmetries

in the MSSM, an $R$ symmetry and a Peccei-Quinn symmetry. This means that one could treat the various dimensionful parameters in (2.1) and (2.2) as spurions which break the symmetries, thus deriving selection rules. The appropriate charge assignments are:

$$
\begin{array}{cccccccccc} 
& m_{\tilde{g}} & A & m_{12}^{2} & \mu & H_{u} & H_{d} & Q \bar{u} & Q \bar{d} & L \bar{\ell} \\
U(1)_{\mathrm{PQ}} & 0 & 0 & -2 & -2 & 1 & 1 & -1 & -1 & -1 \\
U(1)_{\mathrm{R}} & -2 & -2 & -2 & 0 & 1 & 1 & 1 & 1 & 1
\end{array}
$$


Physical observables can only depend on combinations of the dimensionful parameters that are neutral under both $U(1)$ 's. There are three such independent combinations: $m_{\tilde{g}} \mu\left(m_{12}^{2}\right)^{*}, A \mu\left(m_{12}^{2}\right)^{*}$ and $A^{*} m_{\tilde{g}}$. However, only two of their phases are independent, say

$$
\phi_{A}=\arg \left(A^{*} m_{\tilde{g}}\right), \quad \phi_{B}=\arg \left(m_{\tilde{g}} \mu\left(m_{12}^{2}\right)^{*}\right) .
$$

In the more general case of non-universal soft terms there is one independent phase $\phi_{A_{i}}$ for each quark and lepton flavor. Moreover, complex off-diagonal entries in the sfermion mass matrices may represent additional sources of $\mathrm{CP}$ violation.

The most significant effect of $\phi_{A}$ and $\phi_{B}$ is their contribution to electric dipole moments (EDMs). For example, the contribution from one-loop gluino diagrams to the down quark EDM is given by [17-18]:

$$
d_{d}=M_{d} \frac{e \alpha_{3}}{18 \pi \tilde{m}^{4}}\left(\left|A m_{\tilde{g}}\right| \sin \phi_{A}+\tan \beta\left|\mu m_{\tilde{g}}\right| \sin \phi_{B}\right),
$$

where we have taken $m_{Q}^{2} \sim m_{D}^{2} \sim m_{\tilde{g}}^{2} \sim \tilde{m}^{2}$, for left- and right-handed squark and gluino masses. We define, as usual, $\tan \beta=\left\langle H_{u}\right\rangle /\left\langle H_{d}\right\rangle$. Similar one-loop diagrams give rise to chromoelectric dipole moments. The electric and chromoelectric dipole moments of the light quarks $(u, d, s)$ are the main source of $d_{N}$ (the EDM of the neutron), giving [19]

$$
d_{N} \sim 2\left(\frac{100 \mathrm{GeV}}{\tilde{m}}\right)^{2} \sin \phi_{A, B} \times 10^{-23} e \mathrm{~cm}
$$

where, as above, $\tilde{m}$ represents the overall SUSY scale. The present experimental bound, $d_{N}<1.1 \times 10^{-25} \mathrm{ecm}$ [20,21], is then violated for $\mathcal{O}(1)$ phases, unless the masses of superpartners are above $\mathcal{O}(1 \mathrm{TeV})$. Alternatively for light SUSY masses, the new phases should be $<\mathcal{O}\left(10^{-2}\right)$. Notice however that one may consider the actual bound weaker than this, due to the theoretical uncertainty in the estimate of the hadronic matrix elements that lead to eq. (2.6) (see ref. [22] for a recent discussion of possible cancellations among the contributions to $d_{N}$ in the case of a universal $\phi_{A}$ ). With this caveat, whether the phases are small or squarks are heavy, a fine-tuning of order $10^{-2}$ seems to be required, in general, to avoid too large a $d_{N}$. This is the Supersymmetric CP Problem 17, 18,23,24].

In addition to $d_{N}$, the SUSY CP phases contribute to atomic and nuclear EDMs (see a detailed discussion in ref. [19]). The former are also sensitive to phases in the leptonic 
sector. The latter give additional constraints on the quark sector phases. For instance, the bound on the nuclear EDM of ${ }^{199} \mathrm{Hg}$ is comparable to the one given by $d_{N}$. In practice, these additional bounds on SUSY CP phases are not stronger than those from $d_{N}$ (at least in the quark sector). However, since there are significant theoretical uncertainties in the calculation of nuclear EDMs, it is important to measure as many as possible of them to obtain more reliable bounds.

\subsection{The Supersymmetric $\varepsilon_{K}$ Problem}

The contribution to the CP violating $\varepsilon_{K}$ parameter in the neutral $K$ system is dominated by diagrams involving $Q$ and $\bar{d}$ squarks in the same loop 2529]. The corresponding effective four-fermi operator involves fermions of both chiralities, so that its matrix elements are enhanced by $\mathcal{O}\left(m_{K} / m_{s}\right)^{2}$ compared to the chirality conserving operators. For $m_{\tilde{g}} \simeq m_{Q} \simeq m_{D}=\tilde{m}$ (our results depend only weakly on this assumption) and focusing on the contribution from the first two squark families, one gets (we use the results in ref. 29])

$$
\varepsilon_{K}=\frac{5 \alpha_{3}^{2}}{162 \sqrt{2}} \frac{f_{K}^{2} m_{K}}{\tilde{m}^{2} \Delta m_{K}}\left[\left(\frac{m_{K}}{m_{s}+m_{d}}\right)^{2}+\frac{3}{25}\right] \operatorname{Im}\left\{\frac{\left(\delta m_{Q}^{2}\right)_{12}}{m_{Q}^{2}} \frac{\left(\delta m_{D}^{2}\right)_{12}}{m_{D}^{2}}\right\}
$$

where $\left(\delta m_{Q, D}^{2}\right)_{12}$ are the off diagonal entries in the squark mass matrices in a basis where the down quark mass matrix and the gluino couplings are diagonal. These flavor violating quantities are often written as $\left(\delta m_{Q, D}^{2}\right)_{12}=V_{11}^{Q, D} \delta m_{Q, D}^{2} V_{21}^{Q, D *}$, where $\delta m_{Q, D}^{2}$ is the mass splitting among the squarks and $V^{Q, D}$ are the gluino coupling mixing matrices in the mass eigenbasis of quarks and squarks. Note that $\mathrm{CP}$ would be violated even if there were two families only [30]. There are also contributions involving the third family squarks via the (13) and (23) mixings. In some cases the third family contribution actually dominates. Using the experimental value of $\varepsilon_{K}$, we get the constraint

$$
\left(\frac{300 \mathrm{GeV}}{\tilde{m}}\right)^{2}\left|\frac{\left(\delta m_{Q}^{2}\right)_{12}}{m_{Q}^{2}} \frac{\left(\delta m_{D}^{2}\right)_{12}}{m_{D}^{2}}\right| \sin \phi \lesssim 0.5 \times 10^{-7},
$$

where $\phi=\arg \left(\left(\delta m_{Q}^{2}\right)_{12}\left(\delta m_{D}^{2}\right)_{12}\right)$. In a generic supersymmetric framework, we expect $\tilde{m}=\mathcal{O}\left(m_{Z}\right), \delta m_{Q, D}^{2} / m_{Q, D}^{2}=\mathcal{O}(1), V_{i j}^{Q, D}=\mathcal{O}(1)$ and $\sin \phi=\mathcal{O}(1)$. Then the constraint 
(2.8) is generically violated by about seven orders of magnitude. Four-fermi operators with same chirality fermions give a smaller effect. The resulting $\varepsilon_{K}$-bounds are therefore somewhat weaker:

$$
\left(\frac{300 \mathrm{GeV}}{m_{Q}}\right)^{2}\left|\operatorname{Im}\left(\frac{\left(\delta m_{Q}^{2}\right)_{12}}{m_{Q}^{2}}\right)^{2}\right| \lesssim 10^{-6}
$$

and similarly for the right handed squarks.

\subsection{Exact Universality}

Both supersymmetric CP problems are solved if, at the scale $\Lambda_{S}$, the soft supersymmetry breaking terms are universal and the genuine SUSY CP phases $\phi_{A, B}$ vanish. Then the Yukawa matrices represent the only source of flavor and CP violation which is relevant in low energy physics. This situation can naturally arise when supersymmetry breaking is mediated by gauge interactions at a scale $\Lambda_{S} \ll \Lambda_{F}$ 31 34. In the simplest scenarios, the $A$-terms and the gaugino masses are generated by the same SUSY and $U(1)_{R}$ breaking source (see eq. (2.3)). Thus, up to very small effects due to the standard Yukawa matrices, $\arg (A)=\arg \left(m_{\tilde{g}}\right)$ so that $\phi_{A}$ vanishes. In specific models also $\phi_{B}$ vanishes in a similar way 32,34. It is also possible that similar boundary conditions occur when supersymmetry breaking is communicated to the observable sector up at the Planck scale [35]39]. The situation in this case seems to be less under control from the theoretical point of view. Dilaton dominance in SUSY breaking, though, seems a very interesting direction to explore 40,41.

The most important implication of this type of boundary conditions for soft terms, which we refer to as exact universality [42-43], is the existence of the SUSY analogue of the GIM mechanism which operates in the SM. The CP violating phase of the CKM matrix can feed into the soft terms via Renormalization Group ( $R G)$ evolution only with a strong suppression from light quark masses [15].

With regard to the supersymmetric $\mathrm{CP}$ problem, gluino diagrams contribute to quark EDMs as in eq. (2.5), but with a highly suppressed effective phase, e.g.

$$
\phi_{A_{d}} \sim\left(t_{S} / 16 \pi^{2}\right)^{4} Y_{t}^{4} Y_{c}^{2} Y_{b}^{2} J
$$


Here $t_{S}=\log \left(\Lambda_{S} / M_{W}\right)$ arises from the RG evolution from $\Lambda_{S}$ to the electroweak scale, the $Y_{i}$ 's are quark Yukawa couplings (in the mass basis), and $J=\operatorname{Im}\left(V_{u d} V_{t b} V_{u b}^{*} V_{t d}^{*}\right) \simeq 2 \times 10^{-5}$ is the invariant measure of $\mathrm{CP}$ violation in the CKM matrix [44]. A similar contribution comes from chargino diagrams. The resulting EDM is $d_{N} \lesssim 10^{-31} \mathrm{e} \mathrm{cm}$. This maximum can be reached only for very large $\tan \beta \sim 60$ while, for small $\tan \beta \sim 1, d_{N}$ is about 5 orders of magnitude smaller. This range of values for $d_{N}$ is much below the present $\left(\sim 10^{-25} e \mathrm{~cm}\right)$ and foreseen $\left(\sim 10^{-28} e \mathrm{~cm}\right)$ experimental sensitivities. The smallness of these contributions has been recently emphasized in ref. [45], by using the spurionic analysis of ref. [15] which keeps the GIM mechanism manifest. (For previous numerical estimates of the effective phases, see refs. [46-48].)

With regard to the supersymmetric $\varepsilon_{K}$ problem, the contribution to $\varepsilon_{K}$ is proportional to $\operatorname{Im}\left(V_{t d} V_{t s}^{*}\right)^{2} Y_{t}^{4}\left(t_{S} / 16 \pi^{2}\right)^{2}$, giving the same GIM suppression as in the SM. This contribution turns out to be small. Using the result in Ref. [15], we get

$$
\left|\varepsilon_{K}^{\mathrm{SUSY}}\right| \sim 6 \times 10^{-6}\left[\frac{J \operatorname{Re}\left(V_{t d} V_{t s}^{*}\right)}{10^{-8}}\right]\left[\frac{300 \mathrm{GeV}}{\tilde{m}}\right]^{2}\left[\frac{\ln \left(\Lambda_{S} / m_{W}\right)}{5}\right]^{2} .
$$

The value $t_{S}=5$ is typical to gauge mediated supersymmetry breaking, but (2.11) remains negligible for any scale $\Lambda_{S} \lesssim M_{\mathrm{Pl}}$ (namely $t_{S} \lesssim 35$ ). The supersymmetric contribution to $D-\bar{D}$ mixing is similarly small and we expect no observable effects.

For the $B_{d}$ and $B_{s}$ systems, the largest SUSY contribution to the mixing comes from box diagrams with intermediate charged Higgs and the up quarks. It can be up to $\mathcal{O}(0.2)$ of the SM amplitude for $\Lambda_{S}=M_{\mathrm{Pl}}$ and $\tan \beta=\mathcal{O}(1)$ 49, and much smaller for large $\tan \beta$. The contribution is smaller in models of gauge mediated SUSY breaking where the mass of the charged Higgs boson is typically $\gtrsim 300 \mathrm{GeV}$ [33] and $t_{S} \sim 5$. The SUSY contributions to $B_{s} \bar{B}_{s}$ and $B_{d} \bar{B}_{d}$ mixing are, to a good approximation, proportional to $\left(V_{t b} V_{t s}^{*}\right)^{2}$ and $\left(V_{t b} V_{t d}^{*}\right)^{2}$, respectively, like in the SM. Then, regardless of the size of these contributions, the relation $\Delta m_{B_{d}} / \Delta m_{B_{s}} \sim\left|V_{t d} / V_{t s}\right|^{2}$ and the CP asymmetries in neutral $B$ decays into final $\mathrm{CP}$ eigenstates are the same as in the SM.

\subsection{The Non-Universal Case: Approximate CP Symmetry}

Both supersymmetric CP problems are solved if $\mathrm{CP}$ is an approximate symmetry, broken by a small parameter of order $10^{-3}$. This is one of the possible solutions to CP 
problems in the class of supersymmetric models with $\Lambda_{F} \lesssim \Lambda_{S}$, where the soft masses are generically not universal, so that we do not expect flavor and $\mathrm{CP}$ violation to be limited to the Yukawa matrices. 2 Most models where soft terms arise at the Planck scale $\left(\Lambda_{S} \sim M_{\mathrm{Pl}}\right)$ belong to this class.

In order to have a successful mechanism to screen $\mathrm{CP}$ violating phases, a theory or a set of assumptions on the origin of CP violation is needed. Such a theory has to be able to reproduce the only well established evidence of $\mathrm{CP}$ violation in experimental data, $\varepsilon_{K}$, without affecting in an unacceptable way all the other CP odd observables. On this point, supersymmetric models (as many other extensions of the standard model) provide us with two radically different possibilities. A first, perhaps reactionary, (as dubbed in ref. [50] in the context of multi-Higgs models) point of view is that the CKM picture of CP violation is incorrect, i.e. that $\varepsilon_{K} \ll 1$ not because of the smallness of mixing angles and quark mass differences (GIM), but just because CP odd phases happen to be small wherever they appear. In other words, CP is an approximate symmetry of the full theory, not just of the standard model sector. (The second point of view is described in the next two sections.)

If $\mathrm{CP}$ is an approximate symmetry, we expect also the $\mathrm{SM}$ phase $\delta_{\mathrm{KM}}$ to be $\ll 1$. Then the standard box diagrams cannot account for $\varepsilon_{K}$ which should arise from another source. In supersymmetry with non-universal soft terms, the source could be diagrams involving virtual superpartners, mainly squark-gluino box diagrams. Let us call $\left(M_{12}^{K}\right)^{\mathrm{SUSY}}$ the supersymmetric contribution to the $K-\bar{K}$ mixing amplitude. Then the requirements $\operatorname{Re}\left(M_{12}^{K}\right)^{\mathrm{SUSY}} \lesssim \Delta m_{K}$ and $\operatorname{Im}\left(M_{12}^{K}\right)^{\mathrm{SUSY}} \sim \varepsilon_{K} \Delta m_{K}$ imply that the generic CP phases are $\geq \mathcal{O}\left(\varepsilon_{K}\right) \sim 10^{-3}$.

Of course, $d_{N}$ constrains the relevant $\mathrm{CP}$ violating phases to be $\lesssim 10^{-2}$. If all phases are of the same order, then $d_{N}$ must be just below or barely compatible with the present experimental bound. A signal should definitely be found if the accuracy is increased by two orders of magnitude.

The main phenomenological implication of these scenarios is that $\mathrm{CP}$ asymmetries in $B$ meson decays are small, perhaps $\mathcal{O}\left(\varepsilon_{K}\right)$, rather than $\mathcal{O}(1)$ as expected in the SM.

2 Of course, some mechanism has also to suppress the real part of the $\Delta S=2$ amplitude by a sufficient amount. 
Large deviations from the SM are also possible in $\varepsilon^{\prime} / \varepsilon$. Indeed, as can be inferred from ref. [29], when CP violation appears mainly in the diagonal blocks of the squark masssquared matrices, the constraint from $\varepsilon_{K}$ implies $\varepsilon^{\prime} / \varepsilon \lesssim 10^{-5}$. When there is considerable $\mathrm{CP}$ violation also in $A$-terms or gaugino masses $\varepsilon^{\prime} / \varepsilon$ can be larger.

Ref. [52 presents an interesting attempt to naturally generate an approximate CP symmetry: CP is spontaneously broken in a sector of heavy fermions in vector representations of $S U(3) \times S U(2) \times U(1)$, and is transmitted to the MSSM only via radiative corrections. The only resulting observable phases then appear in gaugino masses and are of order $\alpha_{3} / 4 \pi$ for gluinos and $\alpha_{2} / 4 \pi$ for winos. So all CP violating effects are suppressed by $\alpha / 4 \pi$, which seems very promising. 3 However, in order to reproduce the correct value of $\varepsilon_{K}$, this model needs rather large $A$-terms, $A_{12}^{d} \gtrsim 4 Y_{s} \tilde{m}$ (the naive expectation in most flavor models would be $A_{12}^{d} \sim \theta_{C} Y_{s} \tilde{m}$ where $\theta_{C} \sim 0.2$ is the Cabibbo angle). The resulting contribution to EDMs depends on the flavor structure of the $A$-terms and could be large even for small $\phi_{A}$. Such a large $A_{12}^{d}$ also leads to $\varepsilon^{\prime} / \varepsilon \gtrsim 3 \times 10^{-3}$, barely compatible with present bounds.

\subsection{The Non-Universal Case: Approximate Abelian Horizontal Symmetries}

For supersymmetric models with $\Lambda_{F} \lesssim \Lambda_{S}$, where there are genuine supersymmetric sources of flavor and $\mathrm{CP}$ violation, one can still take a point of view that is very different from the one described in the previous section: The CKM picture of CP violation is the correct one, whereby $\varepsilon_{K} \sim 10^{-3}$ results from small flavor mixings rather than small phases in the individual Lagrangian parameters. We now expect $\mathcal{O}(1)$ phases, so that an explanation is needed for the absence or smallness of the new supersymmetric contribution to $\varepsilon_{K}$ and to the EDMs. Therefore, mechanisms to suppress FCNC and to screen the CP phases in the soft SUSY breaking mass terms and in the $A$ terms are required. Abelian horizontal symmetries, which are invoked to explain the flavor structure of the observed quarks, can provide at the same time CP screening mechanisms that are efficient enough to solve both $\mathrm{CP}$ problems.

3 See also ref. [51] which discusses a particular ansatz where CP violation appears only in $A$-terms. 
With regard to the neutral $K$ system, a possible mechanism to screen the CP violating phases in the supersymmetric box diagrams is provided by alignment [53]: The squark mass matrices have a structure, but they have a reason to be diagonal in the basis set by the quark mass matrix. This is achieved in models of Abelian horizontal symmetries [53,54]. The symmetry is spontaneously broken by the VEVs of scalar fields $\{\Phi\}$ ("flavons"), producing a small parameter $\lambda \equiv \Phi / \Lambda_{F}$ which is usually taken to have a value $\lambda \sim 0.2$. This small parameter is responsible for the smallness and hierarchy of quark masses and mixings. The solution of the supersymmetric $\varepsilon_{K}$ problem in this framework makes use of the fact that in supersymmetric theories, the Yukawa matrices $Y^{q}$ must be holomorphic functions of the flavon fields $\{\Phi\}$. By assigning appropriate $\mathcal{H}$-charges to the quark superfields, holomorphy dictates that the $2 \times 2$ down Yukawa sub-matrix is diagonal in the flavor basis, i.e. the basis where the quark fields have definite $\mathcal{H}$ charges. Then the only supersymmetric contributions to $K-\bar{K}$ mixing arise directly via the (12) entries in $m_{Q, D}^{2}$ and, indirectly, via the third family. The left hand side of eq. (2.8) is of order $\lambda^{12} \sim 10^{-8}$, consistent with the bound.

A solution which does not require much universality is also provided by the dynamical mechanism suggested in ref. [55] (see also Ref. [56] for a more critical discussion). There the soft terms correspond to fields that are free to have different orientations in flavor space. Their expectation value is dynamically determined by the only source of "explicit" flavor violation which is assumed to be relevant, the Yukawa matrices. Then all SUSY induced $\mathrm{CP}$ violating effects end up being proportional to $J \sim 2 \times 10^{-5}$. Moreover these effects appear without additional suppression only in operators that do not involve right handed fermions. Thus the leading effect has to satisfy the weaker bound of eq. (2.9) which, with the suppression from $J$, requires only a mild squark degeneracy.

An extension of these ideas, aimed at screening the CP phases in the $A$-terms, is given in Ref. [57]. Since we do not have universality, the phases $\phi_{A}$ are in general different for each quark flavor:

$$
\phi_{A_{q}}=\arg \left(\frac{A_{q} m_{\tilde{g}}^{*}}{Y_{q}}\right) .
$$

(Here, in the definition of $A$, we do not factor out the Yukawa matrices $Y$, as was done instead in the universal case (2.2).) In the model of ref. [57, $\mathrm{CP}$ is assumed to be a 
symmetry of the Lagrangian. The flavon fields $\Phi$ spontaneously break not only $\mathcal{H}$ but also CP. This assumption implies, in particular, that the soft terms, before the breakdown of $\mathcal{H}$, can be all made real. Both the Yukawa matrices and $A$-term matrices are flavon dependent and could be complex through their dependence on $\Phi$. On the other hand, $m_{\tilde{g}}$ is a flavor singlet and therefore real to a very good approximation. A crucial property of supersymmetric theories is that both $A_{q}$ and $Y_{q}$ must be holomorphic functions of the flavon fields. Moreover, since $A_{q}$ and $Y_{q}$ have the same $\mathcal{H}$-charges, the dependence on $\Phi$, apart from different (real) numerical coefficients, must be the same. This is the key point in solving the SUSY CP problem. Consider indeed a simple one flavor case. If the combination of horizontal symmetry and holomorphy allows only one combination of flavon fields $\Phi_{1}$ to contribute to $A$ and $Y$, then we have

$$
Y=y \Phi_{1}, \quad A=m \Phi_{1} \quad \Longrightarrow \quad \phi_{A}=\arg \left(\frac{m}{y}\right)=0
$$

even when $\Phi_{1}$ is complex ( $y$ and $m$ are real parameters according to the assumption of a CP conserving Lagrangian). This mechanism would fail if two combinations of fields, $\Phi_{1}$ and $\Phi_{2}$, contributed:

$$
Y=y_{1} \Phi_{1}+y_{2} \Phi_{2}, \quad A=m_{1} \Phi_{1}+m_{2} \Phi_{2} \quad \Longrightarrow \quad \phi_{A}=\arg \left(\frac{m_{1} \Phi_{1}+m_{2} \Phi_{2}}{y_{1} \Phi_{1}+y_{2} \Phi_{2}}\right) \neq 0
$$

(for $\operatorname{Im}\left(\Phi_{1} \Phi_{2}^{*}\right) \neq 0$ and $y_{1} / y_{2} \neq m_{1} / m_{2}$, which is the general case). This analysis is easily generalized to the relevant case where $A$ and $Y$ are $3 \times 3$ matrices which have complex entries in order to generate a non-zero CKM phase. The result is that SUSY CP phases are suppressed when each eigenvalue in $Y$ is determined to high accuracy by just one combination of operators (also including contributions from off-diagonal terms).

In the model of ref. [57], as a result of the flavor symmetry and holomorphy, the form of the quark Yukawa matrices is approximately triangular. The suppression of multiple contributions to the eigenvalues is mainly due to that. The effective phases are $\mathcal{O}\left(\lambda^{6}\right)$, leading to $d_{N} \sim 10^{-28} e \mathrm{~cm}$.

In models of alignment [53,54], in order to obtain the Cabibbo angle as experimentally measured, it is necessary that the supersymmetric mixing angle between $\tilde{u}_{L}-\tilde{c}_{L}$ is $\mathcal{O}\left(\theta_{C}\right)$. This leads to $D-\bar{D}$ mixing close to the experimental bound. Furthermore, with an 
arbitrary new $\mathrm{CP}$ violating phase in the mixing matrix, interesting $\mathrm{CP}$ violating effects are likely to arise, i.e. a different time dependence between the rates of $D^{0} \rightarrow K^{+} \pi^{-}$and $\bar{D}^{0} \rightarrow K^{-} \pi^{+}$58. It is, however, possible that the mechanism that solves the SUSY CP problems also constrains the new $\mathrm{CP}$ effects in $D-\bar{D}$ mixing to be negligible (as it is indeed the case in the model of ref. 577).

For the neutral $B$ system the relevant supersymmetric mixing angles are suppressed by $\mathcal{O}\left(V_{u b}\right)$. The supersymmetric contribution to $B-\bar{B}$ mixing can be comparable to the SM contribution for squark masses around $300 \mathrm{GeV}$ [54]. The crucial difference with respect to exact universality does not lie, however, in the magnitude of the contributions: these may be too small to be clearly signaled in $\Delta m_{B}$ because of the hadronic uncertainties (most noticeably in $f_{B}$ ). It lies instead in the fact that the phase of the supersymmetric contribution is now generically different from that of the standard $W$-boson box diagrams. Therefore, in models where Abelian flavor symmetries tame the supersymmetric FCNC, large deviations from the SM in CP asymmetries in neutral $B$ decays are possible.

\subsection{The Non-Universal Case: Approximate Non-Abelian Horizontal Symmetries}

In this section, we discuss a mechanism which we call approximate universality, and which is mainly devised to solve (though, in most models, it only relaxes) the $\varepsilon_{K}$ problem. This mechanism is typically associated with models of non-Abelian horizontal symmetries, $\mathcal{H}$, where quarks of the two light families fit into an irreducible doublet. In the flavor basis one expects the splitting among the squarks of these families to be $\mathcal{O}\left(\Phi \Phi^{*} / \Lambda_{F}^{2}\right)$, where $\Phi$ breaks $\mathcal{H}$ and $\Lambda_{F}$ is the flavor scale. The ratio $\lambda^{2}=\Phi \Phi^{*} / \Lambda_{F}^{2}$ is expected to be of the order of some products of CKM mixing angles or light to heavy quark mass ratios [60 (typically $\lambda \sim \theta_{C} \sim 0.2$ ), leading to a suppression of $\varepsilon_{K}$.

Let us discuss in more detail this mechanism by focusing on the $(1,2)$ family sector [61]. In the flavor basis, the $2 \times 2$ Yukawa matrices have the form

$$
Y^{d}=Y_{s}\left(\begin{array}{cc}
y_{11}^{d} & y_{12}^{d} \\
y_{21}^{d} & 1
\end{array}\right), \quad Y^{u}=Y_{c}\left(\begin{array}{cc}
y_{11}^{u} & y_{12}^{u} \\
y_{21}^{u} & 1
\end{array}\right)
$$

One motivation for models of non-Abelian horizontal symmetries is that they can give $y_{12}^{d}=y_{21}^{d} \simeq \theta_{C}$ and $y_{11}^{d} \ll y_{12}^{d} y_{21}^{d}$. Then, since the CKM mixing is mostly generated in the down sector, the well known successful prediction $\left|V_{u s}\right| \simeq \sqrt{m_{d} / m_{s}}$ is generated. 
By phase rotations it is always possible to choose $y_{12}^{d}, y_{21}^{d}$ real, while $y_{11}^{d}$ and $y_{12}^{u}$ are, in general, complex. We define then two $\mathrm{CP}$ violating phases:

$$
\psi_{d}=\frac{\operatorname{Im} y_{11}^{d}}{y_{12}^{d} y_{21}^{d}}, \quad \psi_{u}=\frac{\operatorname{Im} y_{12}^{u}}{y_{12}^{d}} .
$$

The phase $\psi_{d}$ is the phase of the $d$-quark Yukawa coupling, while $\psi_{u}=\arg \left(V_{u s}\right)$. In this convention the (charged current) electroweak hamiltonian for kaon decays is complex with a phase $\psi_{u}$. In the usual convention, where the electroweak hamiltonian is real, $\psi_{u}$ will appear in the $\Delta S=2$ amplitude. We further define the mass-squared splitting between the diagonal entries of the squark mass matrices in the flavor basis,

$$
\delta_{Q, D}=\delta m_{Q, D}^{2} / \tilde{m}^{2}
$$

In most models, the dominant supersymmetric contribution to $\varepsilon_{K}$ is proportional to

$$
\operatorname{Im}\left\{\frac{\left(\delta m_{Q}^{2}\right)_{12}}{m_{Q}^{2}} \frac{\left(\delta m_{D}^{2}\right)_{12}}{m_{D}^{2}}\right\} \simeq y_{12}^{d} y_{21}^{d} \delta_{Q} \delta_{D}\left(\psi_{d}+\psi_{u}\right)
$$

Notice that, generically, $\psi_{d}$ contributes to $d_{N}$, so that we expect $\psi_{d} \lesssim 10^{-2}$ independent of squark splittings. On the other hand, in the simplest cases, $\lambda \sim 0.2$ and $\delta_{Q} \delta_{D} \sim \lambda^{4} \sim 10^{-3}$, so that eq. (2.8) gives a somewhat stronger bound, $\psi_{d} \lesssim 10^{-3}$. Nonetheless, a mechanism to suppress $d_{N}$ will also suppress this contribution to $\varepsilon_{K}$, possibly by a sufficient amount. In particular, there are interesting models with $y_{11}^{u, d}=0$, so that $\psi_{d}=0$. With regard to $\psi_{u}$, the situation is more problematic. Typically, $y_{12}^{u} \sim \sqrt{m_{u} / m_{c}} \sim \lambda^{2}$ with an arbitrary phase, so that $\psi_{u}=\mathcal{O}(\lambda)$. This result is actually unavoidable in the interesting class of models where the (13) and (11) entries of the Yukawa matrices vanish in the flavor basis, leading to the predictions $\left|V_{t d} / V_{t s}\right| \simeq \sqrt{m_{d} / m_{s}}$ and $\left|V_{u b} / V_{c b}\right| \simeq \sqrt{m_{u} / m_{c}}$. To obtain a large CKM phase, a large $\psi_{u}$ is necessary. Consequently, the $\varepsilon_{K}$-bound becomes $\delta_{Q} \delta_{D} \lesssim 10^{-5} \sim \lambda^{7}$. Many simple models (e.g. [59,62]) have $\delta_{Q} \delta_{D} \sim \lambda^{4}$ which relaxes but does not completely solve the $\varepsilon_{K}$ problem. A similar situation holds in other models which do satisfy $\delta_{Q} \delta_{D} \lesssim 10^{-5}$, but which generate somewhat bigger effects via the mixing with the third family [63] or via $\left[\left(\delta m_{Q}^{2}\right)_{12}\right]^{2}$ [64]. There exist however specific models 65-66] where the non-Abelian symmetry does solve the $\varepsilon_{K}$ problem completely. 
We emphasize, nonetheless, that relaxing (without completely solving) the problem is still useful. Essentially all these models would be acceptable if the level of degeneracy were a factor of 10 stronger than the naive expectation from $\mathcal{H}$ selection rules. It has been suggested that a stronger degeneracy may be dynamically induced by RG evolution [39]. Gluino dominance in the squark mass evolution is a possible mechanism, since the contribution of gluino masses through RG evolution is universal. For $m_{Q}^{2} \sim m_{\tilde{g}}^{2}$ at the Planck scale, the gluino contribution to the low energy squark mass-squared dominates the overall original one by a factor of $6 \div 7$. This additional degeneracy is just about what is needed. Other possibilities to completely solve the $\varepsilon_{K}$ problem in these models, without increasing the level of degeneracy, are an approximate CP symmetry (see section 2.4 above) or heavy squarks (see section 2.7 below).

We would like to add a few comments on the SUSY CP problem in models with approximate universality. The same mechanism of screening discussed in the previous section may work for models of non-Abelian symmetries. However, the more constrained form of the Yukawa matrices, and in particular the non-zero (12) and (21) entries, generally leads to a weaker suppression of SUSY phases [61]. Consider for instance the class of models, which includes ref. [57], where the CKM phase originates from some off-diagonal entry in the mass matrix which receives contributions from more than one combination of flavon fields with a relative non-trivial phase. In general, it can be shown that nonuniversality of $A$-terms and the requirement of $\mathcal{O}(1)$ CKM phase imply $\phi_{A} \gtrsim \lambda^{6} \sim J$. The minimal result can be reached only if $Y_{21}$ is highly suppressed (or vanishing), which can be achieved with Abelian flavor symmetries. In models of non-Abelian symmetries, where the two light families are in irreducible doublets, one does not expect this suppression of $Y_{21}$ to hold, so that there are more contributions to the eigenvalues of the light quarks. For example, when $\left|Y_{12}\right| \sim\left|Y_{21}\right| \sim \sqrt{\left|Y_{11} Y_{22}\right|}$, the effective CP phases for light quarks are expected to be $\gtrsim \lambda^{4}$. If, in addition, $\left|Y_{13}\right| \sim\left|Y_{31}\right|$, it is difficult to avoid an effective phase $\sim \lambda^{2}$, i.e. barely compatible with present bounds (see, for example, 67 for a discussion of GUT scenarios).

The situation in supersymmetric models without flavor universality is then very interesting. On one side, we should not be surprised that $d_{N}$ lies below the experimental 
bound. While the models contain new CP violating phases, they also provide a mechanism, directly connected to holomorphy (see eq. (2.13)), to screen the new CP phases. On the other side, since $\delta_{\mathrm{KM}}(\sim 1)$ feeds into SUSY phases in a much less suppressed way than in the MSSM (see eq. (2.10), there should be a non-negligible amount of CP violation in $A$-terms. In a large class of models this leads to $d_{N} \gtrsim 10^{-28} e \mathrm{~cm}$, with the minimum corresponding to $\phi_{A}^{d} \sim \lambda^{6}$ and obtained for specific textures. It is encouraging that this minimal $d_{N}$ seems to be within the reach of the next generation of experiments (see, for example, 68]). While this is about three orders of magnitude below the present experimental bound, it is still a few orders of magnitude above the SM value and, for that matter, the value in the MSSM with exact universality.

In models with approximate universality, the expected size of $D-\bar{D}$ mixing is at least 2-3 orders of magnitude below the present bound. For processes involving the third family, such as $B-\bar{B}$ mixing, non-Abelian models with the third family in a singlet of $\mathcal{H}$ have signatures similar to those of Abelian models. Therefore, similarly to models of alignment, large deviations from the $\mathrm{SM}$ in $\mathrm{CP}$ asymmetries in neutral $B$ decays are possible.

\subsection{Heavy Squarks}

The Supersymmetric CP problem is solved and the $\varepsilon_{K}$ problem is relaxed (but not eliminated) if the masses of the first and second generations squarks $m_{i}$ are larger than the other soft masses, $m_{i}^{2} \sim 100 \tilde{m}^{2}$ [59,60]. This does not necessarily lead to naturalness problems, since these two generations are almost decoupled from the Higgs sector. Explicit models are presented in [59,69,70].

Notice though that, with the possible exception of $m_{\tilde{b}_{R}}^{2}$, third family squark masses cannot naturally be much above $m_{Z}^{2}$. Then for non-zero CP phases in this sector (or for $\phi_{B} \neq 0$ ) one can still generate a sizeable EDM of the neutron via the two-loop induced three-gluon operator [71]. Indeed, for a light right-handed sbottom, the contribution to $d_{N}$ is about 19

$$
d_{N} \sim\left(\frac{100 \mathrm{GeV}}{m_{\tilde{b}}}\right)^{2} \phi_{A_{b}} \times 10^{-24} e \mathrm{~cm} .
$$

For top squarks, naturalness constrains both stops to be light, but their contribution is about an order of magnitude smaller because of the different QCD dressing [72,75]. 
We conclude that, if phases are generically of order one, the main contribution to $d_{N}$ comes from the third family and it is roughly at the present experimental bound when $m_{\tilde{t}_{L, R}} \sim 100 \mathrm{GeV}$.

The upper bound from naturalness on the first two generations is $m_{Q, D} \lesssim 20 \mathrm{TeV}$ for low $\Lambda_{S}$, and even stronger, $2-5 \mathrm{TeV}$, for $\Lambda_{S} \sim M_{\mathrm{Pl}}[76]$. When these bounds are taken into account, eq. (2.8) is not satisfied, in general. Combining this scenario with alignment, $\left(\delta m_{Q, D}^{2}\right)_{12} \sim \sin \theta_{C} \delta m_{Q, D}^{2}$, would solve the $\Delta m_{K}$ problem, but the contribution to $\varepsilon_{K}$ would still be too large, unless

$$
\frac{\delta m_{Q}^{2}}{m_{Q}^{2}} \frac{\delta m_{D}^{2}}{m_{D}^{2}} \sin \phi \lesssim 10^{-2} .
$$

However, this scenario becomes viable when further combined with the approximate universality of the models with non-Abelian horizontal symmetries. All those models which were problematic with light squarks do satisfy the milder eq. (2.20). Notice, though, that the universal contribution to squark masses from gluino terms in the RG evolution cannot play a significant role here. This is because gluino masses affect the stop mass and are thus constrained by naturalness to be around the weak scale.

Models with the first two squark generations heavy have their own signatures of CP violation in neutral meson mixing [77]. The mixing angles relevant to $D-\bar{D}$ mixing are similar, in general, to those of models of alignment (if alignment is invoked to explain $\Delta m_{K}$ with $\left.m_{Q, D}^{2} \lesssim 20 T e V\right)$. However, as $\tilde{u}$ and $\tilde{c}$ squarks are heavy, the contribution to $D-\bar{D}$ mixing is only about one to two orders of magnitude below the experimental bound. This may lead to the interesting situation that $D-\bar{D}$ mixing will first be observed through its $\mathrm{CP}$ violating part $[78$. In the neutral $B$ system, $\mathcal{O}(1)$ shifts from the Standard Model predictions of $\mathrm{CP}$ asymmetries in the decays to final $\mathrm{CP}$ eigenstates are possible. This can occur even when the squarks masses of the third family are $\sim 1 \mathrm{TeV}$ [69], since now mixing angles can naturally be larger than in the case of horizontal symmetries (alignment or approximate universality).

\section{Extensions of the Fermion Sector}

The fermion sector of the Standard Model consists of three generations, with $(i=$ 
$1,2,3)$

$$
Q_{i}(3,2)_{+1 / 6}, \quad \bar{u}_{i}(\overline{3}, 1)_{-2 / 3}, \quad \bar{d}_{i}(\overline{3}, 1)_{+1 / 3}, \quad L_{i}(1,2)_{-1 / 2}, \quad \bar{\ell}_{i}(1,1)_{+1},
$$

representations of the $S U(3) \times S U(2) \times U(1)$ gauge group. It can be extended by either a fourth, sequential generation or by non-sequential fermions, namely 'exotic' representations, different from those of (3.1). . M Most of our discussion in this chapter is focused on non-sequential fermions and their implications on $\mathrm{CP}$ asymmetries in neutral $B$ decays. (For the general formalism of $\mathrm{CP}$ asymmetries in neutral $B$ decays see e.g. 80,82. For model-independent analyses of New Physics effects see [83-88].) The last two sections discuss other processes: $K_{L} \rightarrow \pi \nu \bar{\nu}$ (with non-sequential quarks) and $\Delta \Gamma\left(B_{s}\right)$ (with a sequential fourth generation).

\subsection{The Theoretical Framework}

We consider a model with extra quarks in vector-like representations of the standard Model gauge group,

$$
d_{4}(3,1)_{-1 / 3}+\bar{d}_{4}(\overline{3}, 1)_{+1 / 3},
$$

Such (three pairs of) quark representations appear, for example, in $E_{6}$ GUTs. The most interesting effects in this model concern $\mathrm{CP}$ asymmetries in neutral $B$ decays into final $\mathrm{CP}$ eigenstates 89-95. We describe these effects in detail as they illustrate the type of new ingredients that are likely to affect $\mathrm{CP}$ asymmetries in neutral $B$ decays and the way in which the SM predictions might be modified. 0

The most important feature of this model for our purposes is that it allows CP violating $Z$-mediated Flavor Changing Neutral Currents (FCNC). To understand how these FCNC arise, it is convenient to work in a basis where the up sector interaction eigenstates are

4 The four generation model became rather unlikely in view of the experimental fact that there are only three massless (or light) neutrinos from $L_{i}$ representations. However, if neutrinos acquire their masses from a see-saw mechanism, and if the scale of right-handed neutrino masses is close to the electroweak one, then it is quite possible that a fourth generation neutrino is heavy enough to evade experimental and cosmological constraints [79].

5 If there exist light up quarks in exotic representations, they may introduce similar, interesting effects in neutral $D$ decays 58 . 
identified with the mass eigenstates. The down sector interaction eigenstates are then related to the mass eigenstates by a $4 \times 4$ unitary matrix $K$. Charged current interactions are described by

$$
\begin{aligned}
\mathcal{L}_{\text {int }}^{W} & =\frac{g}{\sqrt{2}}\left(W_{\mu}^{-} J^{\mu+}+W_{\mu}^{+} J^{\mu-}\right), \\
J^{\mu-} & =V_{i j} \bar{u}_{i L} \gamma^{\mu} d_{j L} .
\end{aligned}
$$

The charged current mixing matrix $V$ is a $3 \times 4$ sub-matrix of $K$ :

$$
V_{i j}=K_{i j} \text { for } i=1,2,3 ; j=1,2,3,4 \text {. }
$$

The $V$ matrix is parameterized by six real angles and three phases, instead of three angles and one phase in the original CKM matrix. All three phases may affect CP asymmetries in $B^{0}$ decays. Neutral current interactions are described by

$$
\begin{aligned}
\mathcal{L}_{\text {int }}^{Z} & =\frac{g}{\cos \theta_{W}} Z_{\mu}\left(J^{\mu 3}-\sin ^{2} \theta_{W} J_{\mathrm{EM}}^{\mu}\right), \\
J^{\mu 3} & =-\frac{1}{2} U_{p q} \bar{d}_{p L} \gamma^{\mu} d_{q L}+\frac{1}{2} \delta_{i j} \bar{u}_{i L} \gamma^{\mu} u_{j L} .
\end{aligned}
$$

The neutral current mixing matrix for the down sector is $U=V^{\dagger} V$. As $V$ is not unitary, $U \neq 1$. In particular, its non-diagonal elements do not vanish:

$$
U_{p q}=-K_{4 p}^{*} K_{4 q} \text { for } p \neq q
$$

The three elements which are most relevant to our study are

$$
\begin{aligned}
& U_{d s}=V_{u d}^{*} V_{u s}+V_{c d}^{*} V_{c s}+V_{t d}^{*} V_{t s}, \\
& U_{d b}=V_{u d}^{*} V_{u b}+V_{c d}^{*} V_{c b}+V_{t d}^{*} V_{t b}, \\
& U_{s b}=V_{u s}^{*} V_{u b}+V_{c s}^{*} V_{c b}+V_{t s}^{*} V_{t b} .
\end{aligned}
$$

The fact that, in contrast to the Standard Model, the various $U_{p q}$ do not necessarily vanish, allows FCNC at tree level. This may substantially modify the predictions for CP asymmetries.

\subsection{Implications of $Z$-Mediated $F C N C$}

The flavor changing couplings of the $Z$ contribute to various FCNC processes. Relevant constraints arise from semileptonic FCNC $B$ decays:

$$
\frac{\Gamma(B \rightarrow \bar{\ell} \bar{\ell})_{Z}}{\Gamma(B \rightarrow e \nu X)}=\left[\left(T_{3}-Q \sin ^{2} \theta_{W}\right)^{2}+\left(Q \sin ^{2} \theta_{W}\right)^{2}\right] \frac{\left|U_{d b}\right|^{2}+\left|U_{s b}\right|^{2}}{\left|V_{u b}\right|^{2}+F_{\mathrm{ps}}\left|V_{c b}\right|^{2}},
$$


where $F_{\mathrm{ps}} \sim 0.5$ is a phase space factor, $\ell$ is any of $\nu_{i}(i=1,2,3), e^{-}$or $\mu^{-}, T_{3}=$ $+1 / 2[-1 / 2]$ and $Q=0[-1]$ for $\nu_{i}\left[\ell^{-}\right]$. The experimental upper bounds [96-98 and, in particular, a preliminary D0 result [99], $\mathrm{BR}\left(B \rightarrow X \mu^{+} \mu^{-}\right) \leq 3.6 \times 10^{-5}$, imply then

$$
\left|\frac{U_{d b}}{V_{c b}}\right| \leq 0.04, \quad\left|\frac{U_{s b}}{V_{c b}}\right| \leq 0.04
$$

Additional constraints come from neutral $B$ mixing:

$$
\left(x_{d}\right)_{Z}=\frac{\sqrt{2} G_{F} B_{B} f_{B}^{2} M_{B} \eta_{B} \tau_{b}}{6}\left|U_{d b}\right|^{2} .
$$

The resulting bound is sensitive to the range taken for the poorly known parameter $f_{B}$. It is of order $\left|U_{d b}\right| \lesssim 10^{-3}$ which is comparable to (3.9). As for $x_{s}$, only lower bounds exist and consequently there is no analog bound on $\left|U_{s b}\right|$.

If the $U_{q b}$ elements are not much smaller than the bounds (3.9), they will affect several aspects of physics related to $\mathrm{CP}$ asymmetries in $B$ decays.

(i) Neutral $B$ mixing:

The experimentally measured value of $x_{d}$ (and the lower bound on $x_{s}$ ) can be explained by Standard Model processes, namely box diagrams with intermediate top quarks. Still, the uncertainties in the theoretical calculations, such as the values of $f_{B}$ and $V_{t d}$ (and the absence of an upper bound on $x_{s}$ ) allow a situation where SM processes do not give the dominant contributions to either or both of $x_{d}$ and $x_{s}$. For example, for $m_{t} \approx 180 \mathrm{GeV}$,

$$
\left(x_{d}\right)_{\text {box }}=0.17\left[\frac{\sqrt{B_{B}} f_{B}}{0.14 G e V}\right]^{2}\left[\frac{\tau_{b}\left|V_{c b}\right|^{2}}{3.5 \times 10^{9} G e V^{-1}}\right]\left[\frac{\left|V_{t d} / V_{c b}\right|}{0.12}\right]^{2},
$$

namely, the Standard Model box diagrams could account for as little as $25 \%$ of the experimental value of $x_{d}$, and even less if the unitarity of the CKM matrix does not hold, in which case the lower bound $\left|V_{t d} / V_{c b}\right| \geq 0.12$ can be violated.

The ratio between the $Z$-mediated tree diagram and the Standard Model box diagram is given by $(q=d, s)$

$$
\frac{\left(x_{q}\right)_{\text {tree }}}{\left(x_{q}\right)_{\text {box }}}=\frac{\sqrt{2} \pi^{2}}{G_{F} m_{W}^{2} y_{t} f_{2}\left(y_{t}\right)}\left|\frac{U_{q b}}{V_{t q} V_{t b}^{*}}\right|^{2} \approx 80\left|\frac{U_{q b}}{V_{t q} V_{t b}^{*}}\right|^{2} .
$$

From (3.9) and (3.12) we learn that the $Z$-mediated tree diagram could give the dominant contribution to $x_{d}$ but at most $\mathcal{O}(0.1)$ of $x_{s}$. 
(ii) Unitarity of the $3 \times 3$ CKM matrix.

Within the SM, unitarity of the three generation CKM matrix gives:

$$
\begin{aligned}
& \mathcal{U}_{d s} \equiv V_{u d}^{*} V_{u s}+V_{c d}^{*} V_{c s}+V_{t d}^{*} V_{t s}=0, \\
& \mathcal{U}_{d b} \equiv V_{u d}^{*} V_{u b}+V_{c d}^{*} V_{c b}+V_{t d}^{*} V_{t b}=0 \\
& \mathcal{U}_{s b} \equiv V_{u s}^{*} V_{u b}+V_{c s}^{*} V_{c b}+V_{t s}^{*} V_{t b}=0 .
\end{aligned}
$$

Eq. (3.7), however, implies that now (3.13) is replaced by

$$
\mathcal{U}_{d s}=U_{d s}, \quad \mathcal{U}_{d b}=U_{d b}, \quad \mathcal{U}_{s b}=U_{s b} .
$$

A measure of the violation of $(3.13)$ is given by

$$
\left|\frac{U_{d s}}{V_{u d} V_{u s}^{*}}\right| \leq 5 \times 10^{-4}, \quad\left|\frac{U_{d b}}{V_{t d} V_{t b}^{*}}\right| \leq 0.3, \quad\left|\frac{U_{s b}}{V_{t s} V_{t b}^{*}}\right| \leq 0.04 .
$$

The bound on $\left|U_{d b} / V_{t d} V_{t b}^{*}\right|$ is even weaker if $\left|V_{t d}\right|$ is lower than the three generation unitarity bound. The bound on $\left|U_{d s}\right|$ follows from the experimental values of (or bounds on) $\mathrm{BR}\left(K^{+} \rightarrow \pi^{+} \nu \bar{\nu}\right), \epsilon_{K}$ and $\operatorname{BR}\left(K_{L} \rightarrow \mu^{+} \mu^{-}\right)$that we present later. The first of the SM relations in (3.13) is practically maintained, while the second can be violated by at most $5 \%$. However, the $\mathcal{U}_{d b}=0$ constraint may be violated by $\mathcal{O}(0.3)$ effects. The Standard Model unitarity triangle should be replaced by a unitarity quadrangle. A geometrical representation of the new relation is given in fig. 1.

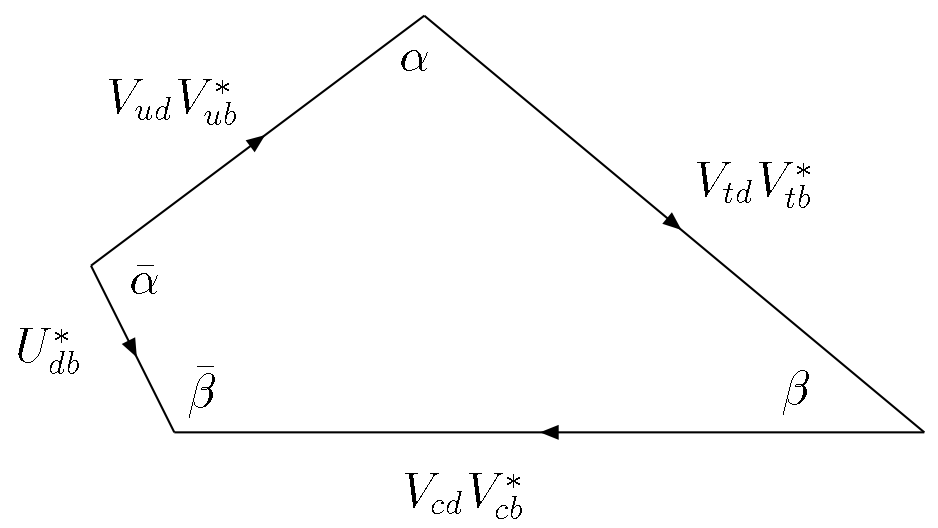

Figure 1. The unitarity quadrangle 
It should be stressed that, at present, only the magnitudes of $U_{d b}$ and $U_{s b}$ are constrained. Each of the phases $\bar{\alpha}$ and $\bar{\beta}$,

$$
\bar{\alpha} \equiv \arg \left(\frac{V_{u d} V_{u b}^{*}}{U_{d b}^{*}}\right), \quad \bar{\beta} \equiv \arg \left(\frac{U_{d b}^{*}}{V_{c d} V_{c b}^{*}}\right),
$$

could be anywhere in the range $[0,2 \pi]$.

(iii) $Z$-mediated $B$ decays.

Our main interest in this chapter is in hadronic $B^{0}$ decays to $\mathrm{CP}$ eigenstates, where the quark sub-process is $\bar{b} \rightarrow \bar{u}_{i} u_{i} \bar{d}_{j}$, with $u_{i}=u, c$ and $d_{j}=d, s$. These decays get new contributions from $Z$-mediated tree diagrams, in addition to the standard $W$-mediated ones. The ratio between the amplitudes is

$$
\frac{A_{Z}}{A_{W}}=\left[\frac{1}{2}-\frac{2}{3} \sin ^{2} \theta_{W}\right]\left|\frac{U_{j b}^{*}}{V_{i j} V_{i b}^{*}}\right| .
$$

We find that the $Z$ contributions can be safely neglected in $\bar{b} \rightarrow \bar{c} c \bar{s}(\lesssim 0.013)$ and $\bar{b} \rightarrow \bar{c} c \bar{d}$ ( $\lesssim 0.06)$. On the other hand, it may be significant in $\bar{b} \rightarrow \bar{u} u \bar{d}(\lesssim 0.25)$, and in processes with no SM tree contributions, e.g. $\bar{b} \rightarrow \bar{s} s \bar{s}$, that may have comparable contributions from penguin and $Z$-mediated tree diagrams.

(iv) New contributions to $\Gamma_{12}\left(B_{q}\right)$

The difference in width comes from modes that are common to $B_{q}$ and $\bar{B}_{q}$. As discussed above, there are new contributions to such modes from $Z$-mediated FCNC. However, while the new contributions to $M_{12}$ are from tree level diagrams, i.e. $\mathcal{O}\left(g^{2}\right)$, those to $\Gamma_{12}$ are still coming form a box-diagram, i.e. $\mathcal{O}\left(g^{4}\right)$. Consequently, no significant enhancement of the SM value of $\Gamma_{12}$ is expected, and the relation $\Gamma_{12} \ll M_{12}$ is maintained.6

\section{3. $C P$ Asymmetries in $B$ Decays}

The fact that $M_{12}\left(B^{0}\right)$ could be dominated by the $Z$-mediated FCNC together with the fact that this new amplitude depends on new $\mathrm{CP}$ violating phases means that large deviations from the Standard Model predictions for $\mathrm{CP}$ asymmetries are possible. As

6 The new contribution could significantly modify the leptonic asymmetry in neutral $B$ decays [100, though the asymmetry remains small. 
$\Gamma_{12} \ll M_{12}$ is maintained, future measurements of certain modes will still be subject to a clean theoretical interpretation in terms of the extended electroweak sector parameters.

Let us assume that, indeed, $M_{12}$ is dominated by the new physics.

$$
\left(\frac{p}{q}\right)_{B} \approx \frac{U_{d b}^{*}}{U_{d b}}
$$

We argued above that $b \rightarrow c \bar{c} s$ is still dominated by the $W$ mediated diagram. Furthermore, the first unitarity constraint in (3.13) is practically maintained. Then it is straightforward to evaluate the CP asymmetry in $B \rightarrow \psi K_{S}$. We find that it simply measures an angle of the unitarity quadrangle of fig. 1 :

$$
a_{C P}\left(B \rightarrow \psi K_{S}\right)=-\sin 2 \bar{\beta} .
$$

The new contribution to $b \rightarrow c \bar{c} d$ is $\mathcal{O}(5 \%)$, which is the same order as the SM penguins and the expected experimental sensitivity. So we still have (taking into account CP-parities)

$$
a_{C P}\left(B \rightarrow \psi K_{S}\right) \approx-a_{C P}(B \rightarrow D D) .
$$

Care has to be taken regarding $b \rightarrow u \bar{u} d$ decays. Here, direct CP violation may be large [95] and prevent a clean theoretical interpretation of the asymmetry. Only if the asymmetry is large, so that the shift from the $Z$-mediated contribution to the decay is small, we get

$$
a_{C P}(B \rightarrow \pi \pi)=-\sin 2 \bar{\alpha} .
$$

The important point about the modification of the SM predictions is then not that the angles $\alpha, \beta$ and $\gamma$ may have very different values from those predicted by the SM, but rather that the $\mathrm{CP}$ asymmetries do not measure these angles anymore. As there are no experimental constraints on $\bar{\alpha}$ and $\bar{\beta}$, the full range $[-1,+1]$ is possible for each of the asymmetries. This model demonstrates that there exist extensions of the SM where dramatic deviations from its predictions for $\mathrm{CP}$ asymmetries in $B$ decays are not unlikely.

7 Generalization to the case that the new contribution is comparable to (but not necessarily dominant over) the Standard Model one is straightforward [91]. 
Another interesting point concerns $B_{s}$ decays. As $B_{s}-\bar{B}_{s}$ mixing as well as the $b \rightarrow c \bar{c} s$ decay are dominated by the SM diagrams, we have, as in the SM,

$$
a_{C P}\left(B_{s} \rightarrow \psi \phi\right) \approx 0
$$

As shown in ref. [83], this is a sufficient condition for the angles extracted from $B \rightarrow \psi K_{S}$, $B \rightarrow \pi \pi$ and $B_{s} \rightarrow \rho K_{S}$ to sum up to $\pi$ (up to possible effects of direct CP violation). This happens in spite of the fact that the first two asymmetries do not correspond to $\beta$ and $\alpha$ of the unitarity triangle.

\subsection{The $K_{L} \rightarrow \pi \nu \bar{\nu}$ Decay}

$K_{L} \rightarrow \pi \nu \bar{\nu}$ is dominated by CP violating effects 101.8 In the SM, the decay amplitude is dominated by top penguin and box diagrams and can be calculated with very little theoretical uncertainties [103]. It then provides a clean measurement of the $\mathrm{CP}$ violating measure $J$ and, together with $K^{+} \rightarrow \pi^{+} \nu \bar{\nu}$, of the angle $\beta$ [104. Studies of New Physics effects on this decay can be found in [105,[106]. Below we present a model independent formalism to analyze this mode and explain why it is a manifestation of $\mathrm{CP}$ violation in interference between mixing and decay (which is the reason for its theoretical cleanliness).

In the neutral $K$ system, the deviation of $|q / p|$ from unity is experimentally measured (by the CP asymmetry in $\left.K_{L} \rightarrow \pi \ell \nu\right)$ and is $\mathcal{O}\left(\operatorname{Re} \varepsilon_{K}\right)$, that is negligibly small for the purposes of this section. For $|q / p|=1$, the time dependence of an untagged sample of $K$ mesons to decay into a $\mathrm{CP}$ eigenstates $f\left(=\pi^{0} \nu \bar{\nu}\right)$ is given by [107]

$$
\Gamma[f(t)]=\frac{\Gamma(K \rightarrow f)}{2}\left\{\left(1+|\lambda|^{2}\right)\left(e^{-\Gamma_{S} t}+e^{-\Gamma_{L} t}\right)+2 \operatorname{Re} \lambda\left(e^{-\Gamma_{S} t}-e^{-\Gamma_{L} t}\right)\right\}
$$

where we define

$$
\lambda \equiv \frac{q}{p} \frac{\bar{A}}{A} .
$$

The amplitude $A(\bar{A})$ is the $K^{0}\left(\bar{K}^{0}\right) \rightarrow f$ transition amplitude. The deviation of the ratio $\bar{A} / A$ from unity is also negligibly small. Since there is only one neutral hadron at the

8 Significant CP conserving contributions can arise if lepton flavor is violated. Otherwise, CP conserving contributions are highly suppressed [102]. 
final state, there is no final state interaction phase. An absorptive phase can come from diagrams with two real intermediate pions, like those arising at higher order in chiral perturbation theory, and is negligibly small [108]. Therefore, it is safe to assume that $|\lambda|=1$ to an excellent approximation, and the leading $\mathrm{CP}$ violating effect is then $\operatorname{Im} \lambda \neq 0$, namely interference between mixing and decay.

Defining $\theta$ to be the relative phase between the $K-\bar{K}$ mixing amplitude and the $s \rightarrow d \nu \bar{\nu}$ decay amplitude, namely $\lambda=e^{2 i \theta}$, we get from (3.23):

$$
\frac{\Gamma\left(K_{L} \rightarrow \pi^{0} \nu \bar{\nu}\right)}{\Gamma\left(K_{S} \rightarrow \pi^{0} \nu \bar{\nu}\right)}=\frac{1-\cos 2 \theta}{1+\cos 2 \theta}=\tan ^{2} \theta .
$$

This ratio measures $\theta$ without any information about the magnitude of the decay amplitudes. In practice, however, it will be impossible to measure $\Gamma\left(K_{S} \rightarrow \pi^{0} \nu \bar{\nu}\right)$. We can use the isospin symmetry relation, $A\left(K^{0} \rightarrow \pi^{0} \nu \bar{\nu}\right) / A\left(K^{+} \rightarrow \pi^{+} \nu \bar{\nu}\right)=1 / \sqrt{2}$, and replace the denominator by the charged kaon mode:

$$
a_{C P} \equiv \frac{\Gamma\left(K_{L} \rightarrow \pi^{0} \nu \bar{\nu}\right)}{\Gamma\left(K^{+} \rightarrow \pi^{+} \nu \bar{\nu}\right)}=r_{i s} \frac{1-\cos 2 \theta}{2}=r_{i s} \sin ^{2} \theta
$$

where $r_{i s}=1.048$ is the isospin breaking factor [109]. The ratio (3.26) may be experimentally measurable, as the relevant branching ratios are $\mathcal{O}\left(10^{-10}\right)$ in the SM and even larger in some of its extensions. It will provide us with a very clean and model independent measurement of the $\mathrm{CP}$ violating phase $\theta$.

New Physics can modify both the mixing and the decay amplitudes. The contribution to the mixing can be of the same order as the SM one. However, $\varepsilon_{K}=\mathcal{O}\left(10^{-3}\right)$ implies that any new contribution to the mixing amplitude carries the same phase as the SM one (to $\left.\mathcal{O}\left(10^{-3}\right)\right)$. On the other hand, the upper bound 110

$$
B R\left(K^{+} \rightarrow \pi^{+} \nu \bar{\nu}\right)<2.4 \times 10^{-9}
$$

which is about 30 times larger than the SM prediction [104], allows New Physics to dominate the decay amplitude (with an arbitrary phase). We conclude that the only potentially significant new contribution to $a_{C P}$ can come from the decay amplitude. This is in contrast to the clean $\mathrm{CP}$ asymmetries in the $B$ system where we expect significant effects of New Physics only in the mixing amplitude. 
$Z$-mediated FCNC provide an explicit example of New Physics that may modify the SM prediction for $a_{C P}$ of eq. (3.26) [102]. Assuming that the $Z$-mediated tree diagram dominates $K \rightarrow \pi \nu \bar{\nu}$, we get [89]

$$
\frac{\Gamma\left(K^{+} \rightarrow \pi^{+} \nu \bar{\nu}\right)}{\Gamma\left(K^{+} \rightarrow \pi^{0} e^{+} \nu\right)}=r_{i s}^{+} \frac{1}{4} \frac{\left|U_{d s}\right|^{2}}{\left|V_{u s}\right|^{2}}, \quad \frac{\Gamma\left(K_{L} \rightarrow \pi^{0} \nu \bar{\nu}\right)}{\Gamma\left(K^{+} \rightarrow \pi^{0} e^{+} \nu\right)}=r_{i s}^{0} \frac{1}{4} \frac{\left|\operatorname{Im} U_{d s}\right|^{2}}{\left|V_{u s}\right|^{2}} .
$$

Here $r_{i s}^{0}=0.944$ and $r_{i s}^{+}=0.901$ are the isospin breaking corrections [109] (so that $r_{i s}=r_{i s}^{0} / r_{i s}^{+}$). The ratio (3.26) measures, in this case, $\sin \theta=\operatorname{Im} U_{d s} /\left|U_{d s}\right|$.

Bounds on the relevant couplings come from $K_{L} \rightarrow \mu^{+} \mu^{-}$(where we take into account uncertainties from long distance contributions 1111 112]), from $K^{+} \rightarrow \pi^{+} \nu \bar{\nu}$ (see (3.8) and (3.27)), and from the measurement of $\varepsilon_{K}$ [89,92]:

$$
\left|\operatorname{Re}\left(U_{d s}\right)\right| \lesssim 2 \times 10^{-5}, \quad\left|U_{d s}\right| \leq 1.0 \times 10^{-4}, \quad\left|\operatorname{Re}\left(U_{d s}\right) \operatorname{Im}\left(U_{d s}\right)\right| \lesssim 1.3 \times 10^{-9} .
$$

We learn that large effects are possible. When $\left|\operatorname{Re}\left(U_{d s}\right)\right|$ and $\left|\operatorname{Im}\left(U_{d s}\right)\right|$ are close to their upper bounds, the branching ratios $B R\left(K^{+} \rightarrow \pi^{+} \nu \bar{\nu}\right)$ and $B R\left(K_{L} \rightarrow \pi^{0} \nu \bar{\nu}\right)$ are both $O\left(10^{-9}\right)$ and $a_{C P}$ of eq. (3.26) is $\mathcal{O}(1)$. Furthermore, as in this case the SM contribution is negligible, the measurement of $B R\left(K^{+} \rightarrow \pi^{+} \nu \bar{\nu}\right)$ determines $\left|U_{d s}\right|$, and with the additional measurement of $B R\left(K_{L} \rightarrow \pi^{0} \nu \bar{\nu}\right), \arg \left(U_{d s}\right)$ is determined as well.

To conclude, in models with lepton flavor conservation, $\frac{\Gamma\left(K_{L} \rightarrow \pi^{0} \nu \bar{\nu}\right)}{\Gamma\left(K^{+} \rightarrow \pi^{+} \nu \bar{\nu}\right)} \neq 0$ signifies CP violation. The value of this ratio provides a clean measurement of a $\mathrm{CP}$ violating phase (possibly coming from New Physics).

\subsection{The $B_{s}$ Width Difference}

In the SM, a large width difference is expected in the $B_{s}$ system [113]116]:

$$
\frac{\Delta \Gamma}{\Gamma} \approx 0.2
$$

New physics can contribute significantly to the mass difference. If this contribution is $\mathrm{CP}$ violating, it leads to a reduction of the width difference [117. Below we explain this general result and give an explicit example: the four generation model.

In general, the width difference is given by (for reviews, see e.g. [80-82])

$$
\Delta \Gamma=\frac{4 \operatorname{Re}\left(M_{12} \Gamma_{12}^{*}\right)}{\Delta M} .
$$


The experimental lower bound $\Delta M / \Gamma>8.8$ [118] implies $\Delta M \gg \Delta \Gamma$ and, consequently, $\left|M_{12}\right| \gg\left|\Gamma_{12}\right|$. Thus, to a very good approximation, $\Delta M=2\left|M_{12}\right|$ and

$$
\Delta \Gamma=2\left|\Gamma_{12}\right| \cos 2 \xi, \quad 2 \xi \equiv \arg \left(-M_{12} \Gamma_{12}^{*}\right)
$$

Under the reasonable assumption that the New Physics does not significantly affect the leading decay processes, $\Gamma_{12}$ is dominated by $b \rightarrow c \bar{c} s$ transitions. Consequently, $2 \xi$ is the relative phase between the mixing amplitude and the $b \rightarrow c \bar{c} s$ decay amplitude. In the SM,

$$
\xi=\beta^{\prime} \equiv \arg \left(-\frac{V_{c s}^{*} V_{c b}}{V_{t s} V_{t b}^{*}}\right) \approx 0,
$$

and then $\cos 2 \xi=1$ to a very high accuracy. With new contributions to the mixing amplitude, non-trivial phases may arise, leading to $\cos 2 \xi<1$. This proves our statement: New $\mathrm{CP}$ violating contributions to the mixing always reduce $\Delta \Gamma$ relative to the $\mathrm{SM}$ prediction.

As already mentioned, $Z$-mediated FCNC cannot contribute significantly to the $B_{s}$ mass difference. However, the effects discussed above might appear if there exists a fourth sequential generation. The CKM matrix is extended to a unitary $4 \times 4$ matrix, which can be parameterized by 6 angles and 3 phases. There are new contributions to $B_{s}$ mixing from box diagrams involving one or two $t^{\prime}$-quarks. There are no experimental constraints that forbid the $t^{\prime}$ contribution to be comparable to or even dominate over the SM one. This is the case if $\left|V_{t^{\prime} b} V_{t^{\prime} s}\right|$ is large.

The relevant effects are related to the modification of the unitarity relation

$$
\sum_{i} V_{i b}^{*} V_{i s}=0
$$

where $i$ runs over all up-type quarks. In the SM, the smallness of $\left|V_{u b}^{*} V_{u s}\right|$ leads to $\beta^{\prime} \approx 0$ $($ see $(3.33))$. With a fourth generation, the $i=c, t, t^{\prime}$ terms in (3.34) can be all of the same order. Then, both the SM phase and the new phase from the $t^{\prime}$ contribution could be large:

$$
\arg \left(\frac{V_{c s}^{*} V_{c b}}{V_{t s} V_{t b}^{*}}\right) \neq 0, \quad \arg \left(\frac{V_{c s}^{*} V_{c b}}{V_{t^{\prime} s} V_{t^{\prime} b}^{*}}\right) \neq 0 .
$$

Consequently, $\cos 2 \xi$ can assume any value and the $B_{s}$ width difference can be significantly smaller than in the SM. Such a reduction is an indication of CP violation: the large SM 
prediction for $\Delta \Gamma$ is based on the fact that the decay width into CP-even final states is larger than into $\mathrm{CP}$-odd final states. When new $\mathrm{CP}$ violating phases appear in the mixing amplitude, then the mass eigenstates can differ significantly from the CP eigenstates, and both mass eigenstates are allowed to decay into the CP-even final states. Consequently, $\Delta \Gamma$ is reduced.

\section{Extensions of the Scalar Sector}

\subsection{Charged Scalar Exchange}

When Natural Flavor Conservation (NFC) is maintained CP violation could arise in charged scalar exchange if there were at least three Higgs doublets [119]. This is also the minimal number of doublets required when $\mathrm{CP}$ breaking is spontaneous only [120]. In this case, $\delta_{\mathrm{KM}}=0$ and all $\mathrm{CP}$ violation comes from the mixing of scalar fields. This model is ruled out, as we show below. It is, of course, still a viable possibility that $\mathrm{CP}$ is explicitly broken, in which case both quark and Higgs mixings provide CP violation.

We investigate a multi Higgs doublet model (with $n \geq 3$ doublets) with NFC and assume that it is a different scalar that couples to the down, up and lepton sectors:

$$
-\mathcal{L}_{Y}=-\frac{\phi_{1}^{+}}{v_{1}} \bar{U} V M_{d}^{\text {diag }} P_{R} D+\frac{\phi_{2}^{+}}{v_{2}} \bar{U} M_{u}^{\text {diag }} V P_{L} D-\frac{\phi_{3}^{+}}{v_{3}} \bar{\nu} M_{\ell} P_{R} \ell+\text { h.c. },
$$

where $V$ is the CKM matrix and $P_{L, R}=\left(1 \mp \gamma_{5}\right) / 2$. We denote the physical charged scalars by $H_{i}^{+}(i=1,2, \ldots, n-1)$, and the would-be Goldstone boson (eaten by the $\left.W^{+}\right)$by $H_{n}^{+}$. We define $K$ to be the matrix that rotates the charged scalars from the interaction- to the mass-eigenbasis. Then the Yukawa Lagrangian in the mass basis (for both fermions and scalars) is

$$
\mathcal{L}_{Y}=\frac{G_{F}^{1 / 2}}{2^{1 / 4}} \sum_{i=1}^{n-1}\left\{H_{i}^{+} \bar{U}\left[Y_{i} M_{u}^{\text {diag }} V P_{L}+X_{i} V M_{d}^{\text {diag }} P_{R}\right] D+H_{i}^{+} \bar{\nu}\left[Z_{i} M_{\ell} P_{R}\right] \ell\right\}+\text { h.c. },
$$

where

$$
X_{i}=-\frac{K_{i 1}^{*}}{K_{n 1}^{*}}, \quad Y_{i}=-\frac{K_{i 2}^{*}}{K_{n 2}^{*}}, \quad Z_{i}=-\frac{K_{i 3}^{*}}{K_{n 3}^{*}} .
$$

$\mathrm{CP}$ violation in the charged scalar sector comes from phases in the mixing matrix for

charged scalars. CP violating effects are largest when the lightest charged scalar is much 
lighter than the heaviest one [121,122]. Here we assume that all but the lightest charged scalar $\left(H_{1}^{+}\right)$effectively decouple from the fermions. Then, $\mathrm{CP}$ violating observables depend on three parameters:

$$
\begin{aligned}
& \frac{\operatorname{Im}\left(X Y^{*}\right)}{m_{H}^{2}} \equiv \frac{\operatorname{Im}\left(X_{1} Y_{1}^{*}\right)}{m_{H_{1}}^{2}} \approx \sum_{i=1}^{n-1} \frac{\operatorname{Im}\left(X_{i} Y_{i}^{*}\right)}{m_{H_{i}}^{2}} \\
& \frac{\operatorname{Im}\left(X Z^{*}\right)}{m_{H}^{2}} \equiv \frac{\operatorname{Im}\left(X_{1} Z_{1}^{*}\right)}{m_{H_{1}}^{2}} \approx \sum_{i=1}^{n-1} \frac{\operatorname{Im}\left(X_{i} Z_{i}^{*}\right)}{m_{H_{i}}^{2}} \\
& \frac{\operatorname{Im}\left(Y Z^{*}\right)}{m_{H}^{2}} \equiv \frac{\operatorname{Im}\left(Y_{1} Z_{1}^{*}\right)}{m_{H_{1}}^{2}} \approx \sum_{i=1}^{n-1} \frac{\operatorname{Im}\left(Y_{i} Z_{i}^{*}\right)}{m_{H_{i}}^{2}}
\end{aligned}
$$

$\operatorname{Im}\left(X Y^{*}\right)$ induces $\mathrm{CP}$ violation in the quarks sector, while $\operatorname{Im}\left(X Z^{*}\right)$ and $\operatorname{Im}\left(Y Z^{*}\right)$ give $\mathrm{CP}$ violation that is observable in semi-leptonic processes.

As mentioned above, there is an interesting question of whether charged scalar exchange could be the only source of CP violation. In other words, we would like to know whether a model of extended scalar sector with spontaneous CP violation and NFC is viable. It is not clear that the model could account for $\varepsilon_{K}$ [123 128]. But if it does, then the charged scalar contribution to $d_{N}$ [129] and, more convincingly, to $\Gamma(b \rightarrow s \gamma)$ [130] are too large. We now explain this point in more detail.

In this framework, neither short distance contributions nor long distance ones from intermediate $2 \pi$ state can produce large enough $\varepsilon_{K}$. One needs to assume that the dominant contribution comes from an intermediate $\eta_{0}$ :

$$
\varepsilon_{K} \approx \frac{e^{i \pi / 4}}{\sqrt{2} \Delta m_{K}} \operatorname{Im} \frac{\left\langle K^{0}|H| \eta_{0}\right\rangle\left\langle\eta_{0}|H| \bar{K}^{0}\right\rangle}{m_{K}-m_{\eta_{0}}} .
$$

To account for the numerical value of $\varepsilon_{K}$, the charged scalar parameters should fulfill 127128

$$
\frac{\operatorname{Im}\left(X Y^{*}\right)}{m_{H}^{2}}\left[\ln \frac{m_{H}^{2}}{m_{c}^{2}}-\frac{3}{2}\right]=0.11 \mathrm{GeV}^{-2} .
$$

With $m_{H} \geq 42 \mathrm{GeV}$, this gives

$$
\operatorname{Im}\left(X Y^{*}\right) \gtrsim 40
$$

A large contribution to the EDM of the neutron $d_{N}$ comes from the EDM of the down quark [129]:

$$
d_{N}^{(d)}=\frac{\sqrt{2} G_{F} m_{d}}{9 \pi^{2}} \operatorname{Im}\left(X Y^{*}\right)\left[\eta_{c}\left|V_{c d}\right|^{2} g\left(m_{c}^{2} / m_{H}^{2}\right)+\eta_{t}\left|V_{t d}\right|^{2} g\left(m_{t}^{2} / m_{H}^{2}\right)\right],
$$


with

$$
g(x)=\frac{x}{(1-x)^{2}}\left[\frac{5 x}{4}-\frac{3}{4}-\frac{1-3 x / 2}{1-x} \ln x\right] .
$$

With some conservative assumptions, and using the lower bound (4.7), we get $d_{N} \gtrsim 2.5 \times$ $10^{-25} e \mathrm{~cm}$, a factor of 2 above the experimental upper bound. An even larger contribution comes through the three gluon operator [131]:

$$
\begin{aligned}
d_{N}^{(g)}=4 \times 10^{-21} e \mathrm{~cm}\left(\frac{\alpha_{s}\left(m_{W}\right)}{\alpha_{s}\left(m_{b}\right)}\right)^{14 / 23} & \left(\frac{\alpha_{s}\left(m_{b}\right)}{\alpha_{s}\left(m_{c}\right)}\right)^{54 / 25}\left(\frac{\alpha_{s}\left(m_{c}\right)}{\alpha_{s}\left(m_{N}\right)}\right)^{54 / 27} \\
& \times\left(\frac{g_{s}\left(m_{N}\right)}{4 \pi}\right)^{3} \operatorname{Im}\left(X Y^{*}\right) F_{3}\left(\frac{m_{t}^{2}}{m_{H}^{2}}\right)
\end{aligned}
$$

where

$$
F_{3}(x)=\frac{x}{4(1-x)^{3}}\left(-3+4 x-x^{2}-2 \ln x\right) .
$$

This result, which suffers from large hadronic uncertainties, seems to be about two orders of magnitude above the bound.

The strongest constraint on $\operatorname{Im}\left(X Y^{*}\right)$, however, comes - somewhat surprisingly - from a CP conserving process, the decay $b \rightarrow s \gamma$ [132]:

$$
\mathrm{BR}(b \rightarrow s \gamma) \leq 4.2 \times 10^{-4}
$$

Within our model, this ratio is given by 129 :

$$
\mathrm{BR}(b \rightarrow s \gamma)=C\left|\eta_{2}+G_{W}\left(x_{t}\right)+\left(|Y|^{2} / 3\right) G_{W}\left(y_{t}\right)+\left(X Y^{*}\right) G_{H}\left(y_{t}\right)\right|^{2}
$$

where

$$
C \equiv \frac{3 \alpha \eta_{1}^{2} \mathrm{BR}\left(B \rightarrow X_{c} \ell \nu\right)}{2 \pi F_{\mathrm{ps}}\left(m_{c}^{2} / m_{b}^{2}\right)} \approx 3 \times 10^{-4}
$$

$F_{\mathrm{ps}} \sim 0.5$ is a phase space factor, $\eta_{1} \sim 0.66$ and $\eta_{2} \sim 0.57$ are QCD correction factors 133, $x_{t}=m_{t}^{2} / m_{W}^{2}, y_{t}=m_{t}^{2} / m_{H}^{2}$, and the expressions for the $G$ functions can be found in [129].

The upper bound on $\operatorname{Im}\left(X Y^{*}\right)$ corresponds to a case where the real part of the new diagrams cancels the Standard Model contributions and the upper bound (4.12) is saturated by the imaginary part of these diagrams 130:

$$
\operatorname{Im}\left(X Y^{*}\right) \lesssim \sqrt{\frac{4.2 \times 10^{-4}}{C}} \frac{1}{G_{H}\left(y_{t}\right)}
$$


For $m_{t} \sim 180 \mathrm{GeV}$, we get

$$
\operatorname{Im}\left(X Y^{*}\right) \lesssim \begin{cases}1.6 & m_{H} \sim \frac{1}{2} m_{Z} \\ 3.2 & m_{H} \sim 2 m_{Z}\end{cases}
$$

The upper bound on $\operatorname{Im}\left(X Y^{*}\right)$ implies that charged scalar exchange can make only a negligible contribution to $\varepsilon_{K}$ and cannot be the only source of CP violation [130]. A detailed investigation shows that, in spite of the fact that charged scalar contributions could, in principle, contribute to $B-\bar{B}$ mixing with new phases, this contribution is numerically small and would modify the Standard Model predictions for CP asymmetries in $B$ decays by no more than $\mathcal{O}(0.02)$ [130]. On the other hand, the contribution to $d_{N}$ can still be close to the experimental upper bound.

\subsection{Transverse Lepton Polarization}

As triple-vector correlation is odd under time-reversal, the experimental observation of such correlation would signal $\mathrm{T}$ and - assuming CPT symmetry - CP violation. 9 The muon transverse polarization in $K \rightarrow \pi \mu \nu$ decays [135-140, and the tau transverse polarization in semileptonic heavy quark decays [141 144] are examples of such observables. The lepton transverse polarization cannot be generated by vector or axial-vector interactions only 137,138, so it is particularly suited for searching for CP violating scalar contributions.

The lepton transverse polarization, $P_{\perp}$, in semileptonic decays is defined as the lepton polarization component along the normal vector of the decay plane,

$$
P_{\perp}=\frac{\vec{s}_{\ell} \cdot\left(\vec{p}_{\ell} \times \vec{p}_{X}\right)}{\left|\vec{p}_{\ell} \times \vec{p}_{X}\right|}
$$

where $\vec{s}_{\ell}$ is the lepton spin three-vector and $\vec{p}_{\ell}\left(\vec{p}_{X}\right)$ is the three-momentum of the lepton (hadron). Experimentally, it is useful to define the integrated CP violating asymmetry

$$
a_{C P} \equiv\left\langle P_{\perp}\right\rangle=\frac{\Gamma^{+}-\Gamma^{-}}{\Gamma^{+}+\Gamma^{-}}
$$

9 It is possible to get non-vanishing T-odd observables even without CP violation (see e.g. 134). Such "fake" asymmetries can arise due to CP conserving unitary phases from final state interactions (FSI). They can be removed by comparing the measurements in two CP conjugate channels. 
where $\Gamma^{+}\left(\Gamma^{-}\right)$is the rate of finding the lepton spin parallel (anti-parallel) to the normal vector of the decay plane.

A measurable non-zero $a_{C P}$ will be a clear signal of new sources of CP violation beyond the SM. The SM predictions and the "fake" asymmetries are much smaller than the current experimental sensitivity 145-148. A non-zero $a_{C P}$ can arise in our model from the interference between the $W$-mediated and the $\mathrm{H}^{+}$-mediated tree diagrams. For strange and bottom quark decays, the asymmetry is given by

$$
a_{C P}=C_{p s} \frac{\operatorname{Im}\left(X Z^{*}\right)}{m_{H}^{2}},
$$

while for charm and top quark decays, it is given by

$$
a_{C P}=C_{p s} \frac{\operatorname{Im}\left(Y Z^{*}\right)}{m_{H}^{2}} .
$$

The $C_{p s}$ factor is different for each decay mode. It depends on the phase space integrals, the form factors and masses involved, and has even a mild dependence on $X Z^{*}$ and $Y Z^{*}$ as they affect the total decay rates.

To find how large can these asymmetries be, we study the bounds on the CP violating parameters. In the down sector, the strongest bound is obtained from the measurement of the inclusive $b \rightarrow X \tau \nu$ decay [149]. At the $2 \sigma$ level it reads 150-153:

$$
\frac{\operatorname{Im}\left(X Z^{*}\right)}{m_{H}^{2}}<0.16 \mathrm{GeV}^{-2} .
$$

For the $K^{+} \rightarrow \pi^{0} \mu^{+} \nu$ decay, (4.21) implies 144,154

$$
a_{C P}\left(K^{+} \rightarrow \pi^{0} \mu^{+} \nu\right) \lesssim 8 \times 10^{-3}
$$

which is close to the current experimental bound [149] $a_{C P}\left(K^{+} \rightarrow \pi^{0} \mu^{+} \nu\right)<1.2 \times 10^{-2}$. Since scalars couple more strongly to heavier fermions, the expected signals are stronger in heavy quark decays. For inclusive $B$ decays, (4.21) implies [143, 155]

$$
a_{C P}(B \rightarrow X \tau \nu) \lesssim 0.3
$$

For exclusive $B$ decays [144, the asymmetries are larger but the theoretical uncertainties are also larger. The allowed asymmetries for decays into muon are suppressed by $m_{\mu} / m_{\tau}$ and, in addition, the muon spin is harder to tag. 
In the up sector, the experimental bound on $\operatorname{Im}\left(Y Z^{*}\right)$ is just the product of the bounds on $Y$ and $Z$ [156]. The strongest bound on $|Y|$ comes from the measurement of $R_{b} \equiv \frac{Z \rightarrow b \bar{b}}{Z \rightarrow \text { hadrons }}$. Requiring that the charged Higgs contribution to $R_{b}$ does not exceed 0.003 , we get

$$
|Y| \lesssim \begin{cases}1.2 & m_{H} \sim \frac{1}{2} m_{Z} \\ 1.6 & m_{H} \sim 2 m_{Z}\end{cases}
$$

The charged Higgs contribution is proportional to $|Y|^{2}$, and the full dependence on $m_{H}$ can be found in [157. The bound on $|Z|$ comes from lepton universality in tau decay:

$$
\frac{|Z|}{m_{H}} \leq 1.7 \mathrm{GeV}^{-1}
$$

For exclusive $D$ decays, (4.24) and (4.25) imply that the allowed asymmetries are $\lesssim \mathcal{O}\left(10^{-2}\right)$ [144]. For top decays, we get

$$
a_{C P}(t \rightarrow b \tau \nu) \lesssim 4 \times 10^{-2} .
$$

Choosing an optimal part of phase space can enhance the signal by a factor of about 5 [143]. Since the $W$ is on shell, several other observables can be constructed for top decays [143].

To conclude: Multi Higgs doublet models can give a measurable signal for transverse lepton polarization in $K, B$ and top decays. Such a signal is a clear indication of New Physics.

\subsection{Flavor Changing Neutral Scalar Exchange}

Natural flavor conservation needs not be exact in models of extended scalar sector 158 [161]. In particular, it is quite likely that the existence of the additional scalars is related to flavor symmetries that explain the smallness and hierarchy in the Yukawa couplings. In this case, the new flavor changing couplings of these scalars are suppressed by the same selection rules as those that are responsible to the smallness of fermion masses and mixing, and there is no need to impose NFC [50,162,[167]. An explicit framework, with Abelian horizontal symmetries, was presented in [168,54]. (For another related study, see [169].) We explain the general idea using these models. 
The simplest model of ref. [168] extends the SM by supersymmetry and by an Abelian horizontal symmetry $\mathcal{H}=U(1)\left(\right.$ or $\left.Z_{N}\right)$. The symmetry $\mathcal{H}$ is broken by a VEV of a single scalar $S$ (to which we attribute charge $\mathcal{H}(S)=-1$ ) that is a singlet of the SM gauge group. Consequently, Yukawa couplings that violate $H$ arise only from nonrenormalizable terms. Defining the relevant sums of $\mathcal{H}$ charges through

$$
\begin{aligned}
& n_{i j}^{d}=\mathcal{H}\left(Q_{i}\right)+\mathcal{H}\left(\bar{d}_{j}\right)+\mathcal{H}\left(\phi_{d}\right), \\
& n_{i j}^{u}=\mathcal{H}\left(Q_{i}\right)+\mathcal{H}\left(\bar{u}_{j}\right)+\mathcal{H}\left(\phi_{u}\right),
\end{aligned}
$$

we find the following form for scalar-fermion couplings:

$$
\mathcal{L}_{Y}=X_{i j}^{d}\left(\frac{S}{M}\right)^{n_{i j}^{d}} Q_{i} \bar{d}_{j} \phi_{d}+X_{i j}^{u}\left(\frac{S}{M}\right)^{n_{i j}^{u}} Q_{i} \bar{u}_{j} \phi_{u}
$$

where $M$ is some high energy scale and $X_{i j}^{d}, X_{i j}^{u}$ are $\mathcal{O}(1)$ complex numbers.10 For a singlet $\operatorname{VEV}\langle S\rangle \ll M$, a small parameter $\lambda=\langle S\rangle / M$ suppresses $\mathcal{H}$ violating terms. More precisely, the effective Yukawa terms,

$$
\mathcal{L}_{Y}^{\mathrm{eff}}=Y_{i j}^{d} Q_{i} \bar{d}_{j} \phi_{d}+Y_{i j}^{u} Q_{i} \bar{u}_{j} \phi_{u}
$$

obey selection rules that can be read from 4.28$)(q=u, d)$ :

$$
Y_{i j}^{q}=\mathcal{O}\left[\lambda^{n_{i j}^{q}}\right]
$$

Note that each Yukawa coupling is proportional, in addition to the suppression factors (4.30), to a complex coefficient of order 1.

The smallness and hierarchy in the quark (and lepton) masses and mixing arises now in a natural way. Explicitly (for $i<j$ ):

$$
\begin{aligned}
\left|V_{i j}\right| & \sim \lambda^{\mathcal{H}\left(Q_{i}\right)-\mathcal{H}\left(Q_{j}\right)}, \\
m\left(d_{i}\right) / m\left(d_{j}\right) & \sim \lambda^{\mathcal{H}\left(Q_{i}\right)-\mathcal{H}\left(Q_{j}\right)+\mathcal{H}\left(\bar{d}_{i}\right)-\mathcal{H}\left(\bar{d}_{j}\right)}, \\
m\left(u_{i}\right) / m\left(u_{j}\right) & \sim \lambda^{\mathcal{H}\left(Q_{i}\right)-\mathcal{H}\left(Q_{j}\right)+\mathcal{H}\left(\bar{u}_{i}\right)-\mathcal{H}\left(\bar{u}_{j}\right)} .
\end{aligned}
$$

10 In the supersymmetric framework, $n_{i j}^{q}<0$ implies $X_{i j}^{q}=0$ due to the holomorphy of the superpotential. 
If, for example, we take $\lambda \sim 0.2$, then the order of magnitude of the three CKM mixing angles and of the six quark masses arise naturally for the following $\mathcal{H}$ charge assignments:

$\begin{array}{cccccc}Q_{1} & Q_{2} & Q_{3} & \bar{d}_{1} & \bar{d}_{2} & \bar{d}_{3} \\ (3) & (2) & (0) & (3) & (2) & (2) \\ \bar{u}_{1} & \bar{u}_{2} & \bar{u}_{3} & & \phi_{d} & \phi_{u} \\ (3) & (1) & (0) & & (0) & (0)\end{array}$

(where we took $\tan \beta=\left\langle\phi_{u}\right\rangle /\left\langle\phi_{d}\right\rangle \sim 1$ ).

The singlet scalar $S$ has flavor changing couplings, $Z_{i j}^{q}(q=u, d ; i, j=1,2,3)$. Their magnitude is related to that of the effective Yukawa couplings $Y_{i j}^{q}$ :

$$
Z_{i j}^{q} \sim \frac{\left\langle\phi_{q}\right\rangle}{\langle S\rangle} Y_{i j}^{q} .
$$

These couplings contribute, for example, to $K-\bar{K}$ mixing proportionally to

$$
Z_{12}^{d} Z_{21}^{d *} \sim \frac{m_{d} m_{s}}{\langle S\rangle^{2}},
$$

where we used (4.30), (4.31) and (4.33) to estimate the magnitude of the flavor changing couplings in terms of known quark parameters.

With arbitrary phase factors in the various $Z_{i j}^{q}$ couplings, the contributions to neutral meson mixing are, in general, $\mathrm{CP}$ violating. In particular, there will be a contribution to $\varepsilon_{K}$ from $\operatorname{Im}\left(Z_{12}^{d} Z_{21}^{d *}\right)$. Requiring that the $S$-mediated tree level contribution does not exceed the experimental value of $\varepsilon_{K}$ gives, for $\mathcal{O}(1)$ phases,

$$
M_{S}\langle S\rangle \gtrsim 1.8 T e V^{2} .
$$

We learn that (for $M_{S} \sim\langle S\rangle$ ) the mass of the $S$-scalar could be as low as $1.5 \mathrm{TeV}$, some 4 orders of magnitude below the bound corresponding to $\mathcal{O}(1)$ flavor changing couplings.

The flavor changing couplings of the $S$-scalar lead also to a tree level contribution to $B-\bar{B}$ mixing proportional to

$$
Z_{13}^{d} Z_{31}^{d *} \sim \frac{m_{d} m_{b}}{\langle S\rangle^{2}} .
$$

This means that, for phases of order 1, the neutral scalar exchange accounts for at most a few percent of $B-\bar{B}$ mixing. This cannot be signaled in $\Delta m_{B}$ (because of the hadronic 
uncertainties in the calculation) but could be signalled (if $\langle S\rangle$ is at the lower bound) in $\mathrm{CP}$ asymmetries in $B^{0}$ decays.

Finally, the contribution to $D-\bar{D}$ mixing, proportional to

$$
Z_{12}^{u} Z_{21}^{u *} \sim \frac{m_{u} m_{c}}{\langle S\rangle^{2}}
$$

is below a percent of the current experimental bound. This is unlikely to be discovered in near-future experiments, even if the new phases maximize the interference effects in the $D^{0} \rightarrow K^{-} \pi^{+}$decay.

To summarize, models with horizontal symmetries naturally suppress flavor changing couplings of extra scalars. There is no need to invoke NFC even for new scalars at the TeV scale. Furthermore, the magnitude of the flavor changing couplings is related to the observed fermion parameters. Typically, contributions from neutral scalars with flavor changing couplings could dominate $\varepsilon_{K}$. If they do, then a signal at the few percent level in $\mathrm{CP}$ asymmetries in neutral $B$ decays is quite likely.

\subsection{Neutral Scalar Exchange in Top Physics}

It is possible that the neutral scalars are mixtures of CP-even and CP-odd scalar fields 170-173, 121-122,158. Such a scalar couples to both scalar and pseudoscalar currents:

$$
\mathcal{L}_{Y}=H_{i} \bar{f}\left(a_{i}^{f}+i b_{i}^{f} \gamma_{5}\right) f
$$

where $H_{i}$ is the physical Higgs boson and $a_{i}^{f}, b_{i}^{f}$ are functions of mixing angles in the matrix that diagonalizes the neutral scalar mass matrix. (Specifically, they are proportional to the components of, respectively, $\operatorname{Re} \phi_{u}$ and $\operatorname{Im} \phi_{u}$ in $H_{i}$.) $\mathrm{CP}$ violation in processes involving fermions is proportional to $a_{i}^{f} b_{i}^{f *}$. The natural place to look for manifestations of this type of CP violation is top physics, where the large Yukawa couplings allow large asymmetries 174 182. Note that unlike our discussion above, the asymmetries here have nothing to do with FCNC processes. Actually, in models with NFC (even if softly broken [158]), the effects discussed here contribute negligibly to $\varepsilon_{K}$ and to $\mathrm{CP}$ asymmetries in $B$ decays. On the other hand, two loop diagrams with intermediate neutral scalar and top quark can induce a CP violating three gluon operator [71,131 that would give $d_{N}$ close to the experimental bound [131, 183 -185]. 


\subsection{The Superweak Scenario}

$\mathrm{CP}$ violation via neutral scalar exchange is the most commonly studied realization of the superweak scenario [186]. The original scenario stated that $\mathrm{CP}$ violation appears in a new $\Delta S=2$ interaction while there is no $\mathrm{CP}$ violation in the SM $\Delta S=1$ transitions. Consequently, the only large observable $\mathrm{CP}$ violating effect is $\varepsilon_{K}$, while $\varepsilon^{\prime} / \varepsilon \sim 10^{-8}$ and EDMs are negligibly small. At present, the idea of "superweak CP violation" refers to many different types of models. There are several reasons for this situation:

(i) The work of ref. [186] was concerned only with $\mathrm{CP}$ violation in $K$ decays. In extending the idea to other mesons, one may interpret the idea in various ways. On one side, it is possible that the superweak interaction is significant only in $K-\bar{K}$ mixing and (apart from the relaxation of the $\varepsilon_{K}$-bounds on the CKM parameters) has no effects on mixing of heavier mesons. On the other extreme, one may take the superweak scenario to imply that $\mathrm{CP}$ violation comes from $\Delta F=2$ processes only for all mesons.

(ii) The scenario proposed in [186] did not employ any specific model. It was actually proposed even before the formulation of the Standard Model. To extend the idea to, for example, the neutral $B$ system, a model is required. Various models give very different predictions for $\mathrm{CP}$ asymmetries in $B$ decays.

(iii) It is rather difficult to achieve the superweak scenario in a natural way. In particular, it is difficult to understand why would CP be a good symmetry in one sector of the theory but not in another. Consequently, in most models that employ approximate symmetries, the CKM phase does not vanish and the resulting CP violation is quite different from the original scenario.

(iv) The question of whether $\mathrm{CP}$ violation can occur in $\Delta F=2$ transitions only is not always well-defined. When discussing $\mathrm{CP}$ violation in the interference of mixing and decay, it is a matter of convention to decide whether to put the $\mathrm{CP}$ violating phases in

$q / p(\Delta F=2)$ or $\bar{A} / A(\Delta F=1)$ or both. The common use of the term 'superweak $\mathrm{CP}$ violation' refers to a situation where $|\bar{A} / A|=1$ and there exists a convention where $\bar{A} / A=1$ for all processes.

If one extends the superweak scenario to the $B$ system by assuming that there is $\mathrm{CP}$ violation in $\Delta b=2$ but not in $\Delta b=1$ transitions, the prediction for $\mathrm{CP}$ asymmetries 
in $B$ decays into final $\mathrm{CP}$ eigenstates is that they are equal for all final states [187-189]. Whether these asymmetries are all small or could be large is model dependent. In addition, the asymmetries in charged $B$ decays vanish.

Various models (or scenarios) that realize the main features of the superweak idea can be found in refs. [190-191,159-160]. As mentioned above, there is a considerable variation in their predictions for $\varepsilon^{\prime} / \varepsilon, d_{N}$ and other quantities. Note, in particular, that neither a measurement of $\varepsilon^{\prime} / \varepsilon$ at the level of $10^{-4}$ nor of $d_{N}$ at the level of $10^{-26} e \mathrm{~cm}$ will unambiguously exclude these models.

\section{Left Right Symmetry}

\subsection{The Theoretical Framework}

We study a specific version of Left-Right Symmetric (LRS) models, where P, C and $\mathrm{CP}$ are symmetries of the Lagrangian that are spontaneously broken 192 197. The electroweak gauge group is $S U(2)_{L} \times S U(2)_{R} \times U(1)_{B-L}$. Left-handed quarks reside in $Q_{L}(2,1)_{1 / 3}$ representations and right-handed ones in $Q_{R}(1,2)_{1 / 3}$. The scalar content of the minimal LRS models is [198] $\Phi(2,2)_{0}, \Delta_{L}(3,1)_{2}$ and $\Delta_{R}(1,3)_{2}$. A model with only minimal scalar sector and spontaneous $\mathrm{CP}$ violation predicts unacceptably large FCNC [199]. To avoid this, one has to add scalar singlets or triplets but these do not affect our analysis. The only specific assumption about the scalar sector that we make is the existence of a single $\Phi$ field. The VEV of $\Phi$ is

$$
\langle\Phi\rangle=\left(\begin{array}{cc}
k & 0 \\
0 & k^{\prime} e^{i \eta}
\end{array}\right) .
$$

The relative phase between $k$ and $k^{\prime}, \eta$, spontaneously breaks CP. In principle, it is the only source of $\mathrm{CP}$ violation. Eventually, there are seven $\mathrm{CP}$ violating phases in the mass eigenbasis. They all vanish when $\eta=0$, but practically they are independent parameters.

The phase $\eta$ appears explicitly in the mixing of the charged gauge bosons:

$$
\begin{aligned}
& W_{1}=\cos \xi W_{L}+e^{-i \eta} \sin \xi W_{R}, \\
& W_{2}=-e^{i \eta} \sin \xi W_{L}+\cos \xi W_{R},
\end{aligned}
$$


where

$$
\xi=\frac{k k^{\prime}}{\left\langle\Delta_{R}^{0}\right\rangle^{2}}
$$

The Yukawa couplings are given by

$$
\mathcal{L}_{Y}=\overline{Q_{L}}\left(A \Phi+B \tau_{2} \Phi^{*} \tau_{2}\right) Q_{R}+\text { h.c. },
$$

where $\tau_{2}$ is the Pauli matrix acting in the $S U(2)_{L}$ or $S U(2)_{R}$ space, $A$ and $B$ are matrices in generation space.

$\mathrm{P}$ symmetry requires that $A$ and $B$ are hermitian; $\mathrm{C}$ symmetry requires that $A$ and $B$ are symmetric; and $\mathrm{CP}$ invariance implies that $A$ and $B$ are real. The mass matrices,

$$
\begin{aligned}
& M_{u}=k A+k^{\prime} e^{-i \eta} B, \\
& M_{d}=k^{\prime} e^{i \eta} A+k B,
\end{aligned}
$$

are symmetric. The symmetry of the matrices implies that

$$
V_{R}=F_{u} V_{L}^{*} F_{d}^{\dagger}
$$

where $V_{L}$ and $V_{R}$ are the charged current mixing matrices for $W_{L}$ and $W_{R}$, respectively, while $F_{u}$ and $F_{d}$ are diagonal unitary matrices:

$$
F_{u}=\operatorname{diag}\left(e^{i \phi_{u}}, e^{i \phi_{c}}, e^{i \phi_{t}}\right) ; \quad F_{d}=\operatorname{diag}\left(e^{i \phi_{d}}, e^{i \phi_{s}}, e^{i \phi_{b}}\right)
$$

On top of the single CP violating phase of the CKM matrix $V_{L}$, there are 5 phase differences in $F_{u}, F_{d}$.

For the purpose of studying new contributions to $\mathrm{CP}$ violation, it is simpler to work in a two generation framework. In this case, $V_{L}$ is real and there are 3 phases in $F_{u}, F_{d}$. We define:

$$
\begin{aligned}
\gamma & =\left(\phi_{c}+\phi_{u}-\phi_{s}-\phi_{d}\right) / 2+\eta, \\
\delta_{1} & =\left(\phi_{c}-\phi_{u}+\phi_{s}-\phi_{d}\right) / 2, \\
\delta_{2} & =\left(\phi_{c}-\phi_{u}-\phi_{s}+\phi_{d}\right) / 2 .
\end{aligned}
$$

Choosing a basis where $V_{L}$ is real and the mixing of $W_{L}-W_{R}$ is real, these phases appear in $V_{R}$ only:

$$
V_{W}=\left(\begin{array}{cc}
c_{\xi} & s_{\xi} \\
-s_{\xi} & c_{\xi}
\end{array}\right), \quad V_{L}=\left(\begin{array}{cc}
c_{\theta} & s_{\theta} \\
-s_{\theta} & c_{\theta}
\end{array}\right), \quad V_{R}=e^{i \gamma}\left(\begin{array}{cc}
e^{-i \delta_{2}} c_{\theta} & e^{-i \delta_{1}} s_{\theta} \\
-e^{i \delta_{1}} s_{\theta} & e^{i \delta_{2}} c_{\theta}
\end{array}\right) .
$$




\subsection{Phenomenological Consequences}

For $\varepsilon_{K}$, the dominant contribution comes from box diagrams with both $W_{L}$ and $W_{R}$ in the loop and from tree level diagrams mediated by the extra Higgs doublet. $W_{L}-W_{R}$ mixing can be safely neglected. The value of $M_{12}(K)$ in this model is 194, 197

$$
\frac{M_{12}^{\mathrm{LRS}}}{M_{12}^{\mathrm{SM}}}=1-e^{i\left(\delta_{2}-\delta_{1}\right)}\left[430 \beta-15 \beta \ln \beta+Q_{H}^{2}\left(11600 \beta_{H}-15 \beta_{H} \ln \beta_{H}\right)\right],
$$

where

$$
\beta=\frac{m_{W_{1}}^{2}}{m_{W_{2}}^{2}}, \quad \beta_{H}=\frac{m_{W_{1}}^{2}}{m_{H^{0}}^{2}}, \quad Q_{H}^{2}=\frac{k^{2}+k^{\prime 2}}{k^{2}-k^{\prime 2}}
$$

and we assumed $m_{H^{0}} \sim m_{A^{0}} \sim m_{H^{+}}$. The factor of 430 was first calculated in ref. [200], and it is the product of three smaller numbers: a factor of 2 since two diagrams contribute, a factor of $4\left[\ln \left(m_{W_{1}}^{2} / m_{c}^{2}\right)-1\right] \sim 28$ from loop integration and a factor of 7.6 due to the Lorentz structure of the relevant matrix operator. The factor of 11600 arises because $H^{0}$ contributes at tree level. The contribution from the LRS diagrams could easily dominate $\varepsilon_{K}$. In order that it does not give a too large contribution, we need

$$
\left|\beta \sin \left(\delta_{1}-\delta_{2}\right)\right| \lesssim 10^{-5}
$$

Note that in order that the real part of the same diagrams does not give a too large $\Delta m_{K}$, we require $m_{W_{2}} \gtrsim 1.7 \mathrm{TeV}$ and $m_{H} \gtrsim 8.8 \mathrm{TeV}$. The bound $\beta \lesssim 1 / 430$ implies

$$
\xi \lesssim 2.2 \times 10^{-3}
$$

The most important LRS contributions to $d_{N}$ arise from quark EDMs. The LRS oneloop diagrams involve $W_{L}-W_{R}$ mixing and $W_{R}-d_{R}-u_{i R}$ vertex, so all phases contribute, but $\left(\gamma+\delta_{1}\right)$ which contributes proportionally to $m_{c}$ is the most important one [201-202]:

$$
d_{N} \approx 1.5 \times 10^{-21} \xi \sin \left(\gamma+\delta_{1}\right) e \mathrm{~cm}
$$

This could easily saturate the experimental bound (even with $\xi$ as small as required by (5.13)). In order not to violate the bound, we need

$$
\left|\xi \sin \left(\gamma+\delta_{1}\right)\right| \lesssim 10^{-4}
$$


There are also contributions to $d_{N}$ through the three gluon operator, but these are about an order of magnitude smaller [203].

The LRS contribution to $\varepsilon^{\prime} / \varepsilon$, through tree diagrams involving $W_{L}-W_{R}$ mixing, gives $192,193,202$

$$
\left|\varepsilon^{\prime} / \varepsilon\right| \approx 276 \xi\left|\sin \left(\gamma-\delta_{2}\right)+\sin \left(\gamma-\delta_{1}\right)\right|
$$

This could easily saturate the experimental bound (even with $\xi$ as small as required by (5.13)). In order not to violate the bound, we need

$$
\xi\left|\sin \left(\gamma-\delta_{1}\right)+\sin \left(\gamma-\delta_{1}\right)\right| \lesssim 10^{-5}
$$

The effect of LRS on CP asymmetries in $B$ decays 204 205 is very small, because LRS contributions to $B-\bar{B}$ mixing are small in magnitude. The reason for that is as follows. One of the enhancement factors for the LRS contribution to $K-\bar{K}$ mixing is the hadronic matrix element,

$$
\frac{\left\langle K^{0}\left|\bar{d}_{L} s_{R} \bar{d}_{R} s_{L}\right| \bar{K}^{0}\right\rangle}{\left\langle K^{0}\left|\left(\bar{d}_{L} \gamma^{\mu} s_{L}\right)^{2}\right| \bar{K}^{0}\right\rangle}=\frac{3}{4}\left[\left(\frac{m_{K}}{m_{s}+m_{d}}\right)^{2}+\frac{1}{6}\right] \approx 7.6 .
$$

However, as $m_{B} \approx m_{b}$, there is no similar enhancement in the $B$ system. This implies that if LRS contributions to $K-\bar{K}$ mixing are as large as the Standard Model ones, then the LRS contributions to $B-\bar{B}$ mixing are $\mathcal{O}(0.1)$ of the Standard Model ones.

Finally, we mention that LRS effects on transverse lepton polarization are negligible. This is due to the general result that vector interactions alone cannot give any transverse lepton polarization [137]. Other CP violating observables can be constructed with potentially large effects. For this, an extra independent vector has to be measured. Examples are four body semileptonic kaon decay [138], and $B$ and $D$ decays into vector mesons where the polarization of the vector meson is measured [144]. The current bounds on the model parameters, however, imply that these asymmetries are small [144].

To summarize: even though all the phases in the LRS model with spontaneously broken CP arise from a single phase $\eta$ in the $\operatorname{VEV}\langle\Phi\rangle$, it is difficult to relate their values unless one makes additional assumptions. Thus, the three bounds that we found, (5.12), (5.15) and (5.17), could all be saturated simultaneously [206]. However, without (at least 
mild) fine-tuning, saturation of the $\varepsilon^{\prime} / \varepsilon$ bound would imply that the contribution to $d_{N}$ is two orders of magnitude below the present experimental limit. If $k^{\prime} / k \lesssim 0.1$ and all phases

are of the same order of magnitude, then the $\varepsilon_{K}$ bound is the strongest. If, furthermore, $k^{\prime} / k \ll m_{s} / m_{c}$, then $\varepsilon^{\prime} / \varepsilon$ and $d_{N}$ are related [193,202] through

$$
\left|d_{N}\right|=3.6 \times 10^{-24}\left|\varepsilon^{\prime} / \varepsilon\right| e \mathrm{~cm} .
$$

Finally, no interesting effects on $B-\bar{B}$ mixing are expected.

\section{Conclusions}

In this review, we studied various extensions of the Standard Model and presented the new $\mathrm{CP}$ violating effects that are most likely to occur in these extensions. When thinking of future measurements of CP violating effects as a tool to discover New Physics, we should distinguish between three classes of quantities:

(i) Observables with small theoretical uncertainties. Here, New Physics effects can be unambiguously observed even if they are comparable in magnitude or somewhat smaller than the Standard Model contribution. The observables in this class are mostly manifestations of interference between mixing and decay in neutral meson decays.

- $\mathrm{CP}$ asymmetries in specific $B$-meson decays such as $B_{d} \rightarrow \psi K_{S}, B_{d} \rightarrow \pi \pi$ (with isospin analysis) and $B_{s} \rightarrow \psi \phi$. These asymmetries are sensitive to extensions of the quark sector, i.e. extra quarks in vector-like representations or a fourth generation and to supersymmetric models where FCNC are suppressed by alignment or by heaviness of the first two squark generations.

- The decay rate $K_{L} \rightarrow \pi \nu \nu$. This mode is also sensitive to extensions of the quark sector.

(ii) Observables which are negligibly small (compared to the experimental bound) in the Standard Model. New Physics effects will be signaled if they are much larger than the Standard Model contributions.

- Electric dipole moments. In particular, the electric dipole moments of the neutron and the electron are likely to be close to the experimental bound in supersymmetric models and in various extensions of the Higgs sector. 
- Transverse lepton polarization. These quantities cannot arise from vector interactions only and therefore are a sensitive probe of extensions of the scalar sector.

- CP violation in $D-\bar{D}$ mixing. These effects will test the alignment mechanism to suppress FCNC in supersymmetry. In addition, they might arise in extensions of the quark sector and of the scalar sector.

(iii) Observables with large hadronic uncertainties that are not negligibly small in the Standard Model. The observables in this class are mostly related to CP violation in decay. Beyond their usefulness to improve our understanding of the relevant hadronic aspects, they may also exclude models of New Physics that predict vanishingly small effects.

- $\varepsilon^{\prime} / \varepsilon$.

- Most CP asymmetries in charged $B$ decays.

The coming years hold great promise in clarifying the various puzzles of CP violation. This may well turn to be the leading direction in the search of New Physics.

\section{Acknowledgements}

We thank Helen Quinn for useful comments on the manuscript. Our understanding of CP violation has benefitted from collaborations with Guy Blaylock, Claudio Dib, Lance Dixon, Michael Dine, Isi Dunietz, Fred Gilman, Howard Haber, Haim Harari, Miriam Leurer, Zoltan Ligeti, Zvi Lipkin, David London, Enrico Nardi, Helen Quinn, Uri Sarid, Nati Seiberg, Abe Seiden, Yuri Shirman, Dennis Silverman, Art Snyder and Mihir Worah. Y.G. is supported by the Department of Energy under contract DE-AC03-76SF00515. Y.N. is supported in part by the United States - Israel Binational Science Foundation (BSF), by the Israel Science Foundation, and by the Minerva Foundation (Munich). 


\section{References}

[1] J.H. Christenson, J.W. Cronin, V.L. Fitch and R. Turlay, Phys. Rev. Lett. 13 (1964) 138.

[2] M. Kobayashi and T. Maskawa, Prog. Theo. Phys. 49 (1973) 652.

[3] A.B. Carter and A.I. Sanda, Phys. Rev. Lett. 45 (1980) 952; Phys. Rev. D23 (1981) 1567.

[4] A.D. Sakharov, ZhETF Pis. Red. 5 (1967) 32; JETP Lett. 5 (1967) 24.

[5] G.R. Farrar and M.E. Shaposhnikov, Phys. Rev. D50 (1994) 774.

[6] M.B. Gavela et al., Nucl. Phys. B430 (1994) 382.

[7] P. Huet and E. Sather, Phys. Rev. D51 (1995) 379.

[8] A.G. Cohen, D.B. Kaplan and A.E. Nelson, Ann. Rev. Nucl. Part. Sci. 43 (1993) 27.

[9] J. Ellis, M.K. Gaillard, D.V. Nanopoulos and S. Rudaz, Phys. Lett. 99B (1981) 101.

[10] P. Huet and A.E. Nelson, Phys. Lett. B355 (1995) 229.

[11] H.P. Nilles, Phys. Rep. 110 (1984) 1.

[12] H.E. Haber and G.L. Kane, Phys. Rep. 117 (1985) 75.

[13] R. Barbieri, Riv. Nuovo Cim. 11 (1988) 1.

[14] H.E. Haber, SCIPP 92/33, Lectures given at TASI 92.

[15] M. Dugan, B. Grinstein and L.J. Hall, Nucl. Phys. B255 (1985) 413.

[16] S. Dimopoulos ans S. Thomas, Nucl. Phys. B465 (1996) 23.

[17] W. Buchmuller and D. Wyler, Phys. Lett. B121 (1983) 321.

[18] J. Polchinski and M. Wise, Phys. Lett. B125 (1983) 393.

[19] W. Fischler, S. Paban and S. Thomas, Phys. Lett. B289 (1992) 373.

[20] K.F. Smith et al., Phys. Lett. B234 (1990) 191.

[21] I.S. Altarev et al., Phys. Lett. B276 (1992) 242.

[22] J. Ellis and R. Flores, Phys. Lett. B377 (1996) 83.

[23] J. Ellis, S. Ferrara and D. Nanopoulos, Phys. Lett. B114 (1982) 231.

[24] S. Barr, Int. J. Mod. Phys. A8 (1993) 209.

[25] J. Donoghue, H. Nilles and D. Wyler, Phys. Lett. B128 (1983) 55.

[26] F. Gabbiani and A. Masiero, Nucl. Phys. B322 (1989) 235.

[27] J.S. Hagelin, S. Kelley and T. Tanaka, Nucl. Phys. B415 (1994) 293.

[28] E. Gabrielli, A. Masiero and L. Silvestrini, Phys. Lett. B374 (1996) 80.

[29] F. Gabbiani, E. Gabrielli, A. Masiero and L. Silvestrini, Nucl. Phys. B477 (1996) 321.

[30] Y. Nir, Nucl. Phys. B273 (1986) 567.

[31] M. Dine and A. Nelson, Phys. Rev. D48 (1993) 1277.

[32] M. Dine and A. Nelson and Y. Shirman, Phys. Rev. D51 (1994) 1362.

[33] M. Dine, A. Nelson, Y. Nir and Y. Shirman, Phys. Rev. D53 (1996) 2658.

[34] M. Dine, Y. Nir and Y. Shirman, Phys. Rev. D55 (1997) 1501. 
[35] A.H. Chamseddine, R. Arnowitt and P. Nath, Phys. Rev. Lett. 49 (1982) 970; Nucl. Phys. B227 (1983) 1219.

[36] R. Barbieri, S. Ferrara and C.A. Savoy, Phys. Lett. B119 (1982) 343.

[37] L. Hall, J. Lykken and S. Weinberg, Phys. Rev. D27 (1983) 235.

[38] J. Ellis, C. Kounnas and D.V. Nanopoulos, Nucl. Phys. B247 (1984) 373.

[39] M. Lanzagorta and G.G. Ross, Phys. Lett. B364 (1995) 163.

[40] V. Kaplunovsky and J. Louis, Phys. Lett. B306 (1993) 269

[41] R. Barbieri, J. Louis and M. Moretti, Phys. Lett. B312 (1993) 451; (E) ibid. B316 (1993) 632.

[42] S. Dimopoulos and H. Georgi, Nucl. Phys. B193 (1981) 150.

[43] N. Sakai, Z. Phys. C11 (1981) 153.

[44] C. Jarlskog, Phys. Rev. Lett. 55 (1985) 1039.

[45] A. Romanino and A. Strumia, hep-ph/9610485.

[46] S. Bertolini and F. Vissani, Phys. Lett. B324 (1994) 164.

[47] T. Inui, Y. Mimura, N. Sakai and T. Sasaki, Nucl. Phys. B449 (1995) 49.

[48] S.A. Abel, W.N. Cottingham and I.B. Wittingham, Phys. Lett. B370 (1996) 106.

[49] S. Bertolini, F. Borzumati, A. Masiero and G. Ridolfi, Nucl. Phys. B353 (1991) 591.

[50] L.J. Hall and S. Weinberg, Phys. Rev. D48 (1993) R979.

[51] S.A. Abel and J.M. Frere, Phys. Rev. D55 (1997) 1623.

[52] K.S. Babu and S.M. Barr, Phys. Rev. Lett. 72 (1994) 2831.

[53] Y. Nir and N. Seiberg, Phys. Lett. B309 (1993) 337.

[54] M. Leurer, Y. Nir and N. Seiberg, Nucl. Phys. B420 (1994) 468.

[55] S. Dimopoulos, G.F. Giudice and N. Tetradis, Nucl. Phys. B454 (1995) 59.

[56] R. Rattazzi and U. Sarid, Nucl. Phys. B475 (1996) 27.

[57] Y. Nir and R. Rattazzi, Phys. Lett. B382 (1996) 363.

[58] G. Blaylock, A. Seiden and Y. Nir, Phys. Lett. B355 (1995) 555.

[59] A. Pomarol and D. Tommasini, Nucl. Phys. B466 (1996) 3.

[60] M. Dine, A. Kagan and R.G. Leigh, Phys. Rev. D48 (1993) 4269.

[61] Y. Nir and R. Rattazzi, CERN-TH/96-350 (1996).

[62] P. Pouliot and N. Seiberg, Phys. Lett. B318 (1993) 169.

[63] R. Barbieri, G. Dvali and L.J. Hall, Phys. Lett. B377 (1996) 76.

[64] L.J. Hall and H. Murayama, Phys. Rev. Lett. 75 (1995) 3985.

[65] C. Carone, L.J. Hall and H. Murayama, Phys. Rev. D54 (1996) 2328.

[66] R. Barbieri, L.J. Hall, S. Raby and A. Romanino, hep-ph/9610449.

[67] R. Barbieri, L.J. Hall and A. Strumia, Nucl Phys. B449 (1995) 437.

[68] R. Golub and K. Lamoreaux, Phys. Rep. 237 (1994) 1.

[69] A.G. Cohen, D.B. Kaplan and A.E. Nelson, Phys. Lett. B388 (1996) 588.

[70] G. Dvali and A. Pomarol, CERN-TH-96-192, hep-ph/9607383.

[71] S. Weinberg, Phys. Rev. Lett. 63 (1989) 2333. 
[72] E. Braaten, C.S. Li and T.C. Yuan, Phys. Rev. Lett. 64 (1990) 1709.

[73] G. Boyd, A.K. Gupta, S.P. Trivedi anf M.B. Wise, Phys. Lett. B241 (1990) 584.

[74] J. Dai, H. Dykstra, R.G. Leigh, S. Paban and D. Dicus, Phys. Lett. B237 (1990) 216, (E) B242 (1990) 547.

[75] M. Dine and W. Fischler, Phys. Lett. B242 (1990) 239.

[76] S. Dimopoulos and G.F. Giudice, Phys. Lett. B357 (1995) 573.

[77] A.G. Cohen, D.B. Kaplan, F. Lepeintre and A.E. Nelson, Phys. Rev. Lett. 78 (1997) 2300 .

[78] L. Wolfenstein, Phys. Rev. Lett. 75 (1995) 2460.

[79] H. Harari and Y. Nir, Nucl. Phys. B292 (1987) 251.

[80] Y. Nir, Lectures presented in the 20th SLAC Summer Institute, SLAC-PUB-5874 (1992).

[81] Y. Nir and H.R. Quinn, Ann. Rev. Nucl. Part. Sci. 42 (1992) 211.

[82] I. Dunietz, Ann. Phys. 184 (1988) 350.

[83] Y. Nir and D. Silverman, Nucl. Phys. B345 (1990) 301.

[84] Y. Nir and H.R. Quinn, Phys. Rev. D42 (1990) 1473.

[85] C.O. Dib, D. London and Y. Nir, Int. J. Mod. Phys. A6 (1991) 1253.

[86] N.G. Deshpande, B. Dutta and S. Oh, Phys. Rev. Lett. 77 (1996) 4499.

[87] J.P. Silva and L. Wolfenstein, hep-ph/9610208.

[88] Y. Grossman and M.P. Worah, Phys. Lett. B395 (1997) 241.

[89] Y. Nir and D. Silverman, Phys. Rev. D42 (1990) 1477.

[90] D. Silverman, Phys. Rev. D45 (1992) 1800.

[91] G.C. Branco, T. Morozumi, P.A. Parada and M.N. Rebelo, Phys. Rev. D48 (1993) 1167.

[92] W-S. Choong and D. Silverman, Phys. Rev. D49 (1994) 2322.

[93] V. Barger, M.S. Berger and R.J.N. Phillips, Phys. Rev. D52 (1995) 1663.

[94] D. Silverman, Int. J. Mod. Phys. A11 (1996) 2253.

[95] M. Gronau and D. London, Phys. Rev. D55 (1997) 2845.

[96] C. Albajar et al., UA1 Collaboration, Phys. Lett. B262 (1991) 163.

[97] Y. Grossman, Z. Ligeti and E. Nardi, Nucl. Phys. B465 (1996) 369, (E) B480 (1996) 753.

[98] ALEPH Collaboration, Report no. PA10-019, presented at the 28th International Conference on High Energy Physics, Warsaw, Poland (1996).

[99] D0 collaboration, Report no. FERMILAB-CONF-96/253-E, presented at the 28th International Conference on High Energy Physics, Warsaw, Poland (1996).

[100] G.C. Branco, P.A. Parada and T. Morozumi, Phys. Lett. B306 (1993) 398.

[101] L.S. Littenberg, Phys. Rev. D39 (1989) 3322.

[102] Y. Grossman and Y. Nir, SLAC-PUB-7380, hep-ph/9701313.

[103] A.J. Buras, Phys. Lett. B333 (1994) 476. 
[104] G. Buchalla and A.J. Buras, Phys. Rev. D54 (1996) 6782.

[105] G. Belanger, C.Q. Geng, and P. Turcotte, Phys. Rev. D46 (1992) 2950.

[106] C.E. Carlson, G.D. Dorata and M. Sher, Phys. Rev. D54 (1996) 4393.

[107] I. Dunietz, Phys. Rev. D52 (1995) 3048.

[108] C.Q. Geng, I.J. Hsu and Y.C. Lin, Phys. Lett. B355 (1995) 569.

[109] W. Marciano and Z. Parsa, Phys. Rev. D53 (1996) 1.

[110] S. Adler et al., BNL 787 Collaboration, Phys. Rev. Lett. 76 (1996) 1421.

[111] P. Ko, Phys. Rev. D45 (1992) 174.

[112] G. Buchalla and A.J. Buras, Nucl. Phys. B412 (1994) 106.

[113] J.S. Hagalin, Nucl. Phys. B193 (1981) 123.

[114] M.B. Voloshin, N.G. Uraltsev, V.A. Khoze and M.A. Shifman, Sov. J. Nucl. Phys. 46, (1987) 112.

[115] R. Aleksan, A. Le Yaouanc, L. Oliver, O. Pène and J.-C. Raynal, Phys. Lett. B316 (1993) 567.

[116] M. Beneke, G. Buchalla and I. Dunietz, Phys. Rev. D54 (1996) 4419.

[117] Y. Grossman, Phys. Lett. B380 (1996) 99.

[118] D. Buskulic et al., ALEPH Collaboration, Phys. Lett. B356 (1995) 409.

[119] S. Weinberg, Phys. Rev. Lett. 37 (1976) 657.

[120] G.C. Branco, Phys. Rev. Lett. 44 (1980) 504.

[121] S. Weinberg, Phys. Rev. D42 (1990) 860.

[122] L. Lavoura, Int. J. Mod. Phys. A8 (1993) 375.

[123] A.I. Sanda, Phys. Rev. D23 (1981) 2647.

[124] N.G. Deshpande, Phys. Rev. D23 (1981) 2654.

[125] J.F. Donoghue and B.R. Holstein, Phys. Rev. D32 (1985) 1152.

[126] H.Y. Cheng, Phys. Rev. D34 (1986) 1397.

[127] I.I. Bigi and A.I. Sanda, Phys. Rev. Lett. 58 (1987) 1605.

[128] H.Y. Cheng, Phys. Rev. D42 (1990) 2329.

[129] P. Krawczyk and S. Pokorski, Nucl. Phys. B364 (1991) 11.

[130] Y. Grossman and Y. Nir, Phys. Lett. B313 (1993) 126.

[131] D. Dicus, Phys. Rev. D41 (1990) 999.

[132] M.S. Alam et al., CLEO Collaboration, Phys. Rev. Lett. 74 (1995) 2885.

[133] B. Grinstein, R. Springer and M. Wise, Nucl. Phys. B339 (1990) 269.

[134] M.B. Gavela et al., Phys. Rev. D39 (1989) 1870

[135] T.D. Lee and C.S. Wu, Ann. Rev. Nucl. Sci. 16 (1966) 471.

[136] H.-Y. Cheng, Phys. Rev. D28 (1983) 150.

[137] M. Leurer, Phys. Rev. Lett. 62 (1989) 1967.

[138] P. Castoldi, J.M. Frère and G. Kane, Phys. Rev. D39 (1989) 2633.

[139] R. Garisto and G. Kane, Phys. Rev. D44 (1991) 2038.

[140] G. Bélanger and C.Q. Geng, Phys. Rev. D44 (1991) 2789. 
[141] H.-Y. Cheng, Phys. Rev. D26 (1982) 143.

[142] E. Golowich and G. Valencia, Phys. Rev. D40 (1989) 112.

[143] D. Atwood, G. Eilam and A. Soni, Phys. Rev. Lett. 71 (1993) 492.

[144] R. Garisto, Phys. Rev. D51 (1995) 1107.

[145] E.S. Ginsberg and J. Smith, Phys. Rev. D8 (1973) 3887.

[146] A.R. Zhitnitskii, Yad. Fiz. 31 (1980) 1024 [Sov. J. Nucl. Phys. 31 (1980) 529].

[147] G.S. Adkins, Phys. Rev. D28 (1983) 2885.

[148] J.G. Körner, K. Schilcher and Y.L. Wu, Phys. Lett. B242 (1990) 119.

[149] R.M. Barnett et al., Phys. Rev. D54 (1996) 1.

[150] P. Krawczyk and S. Pokorski, Phys. Rev. Lett. 60 (1988) 182.

[151] J. Kalinowski, Phys. Lett. B245 (1990) 201.

[152] Y. Grossman and Z. Ligeti, Phys. Lett. B332 (1994) 373.

[153] Y. Grossman, H.E. Haber and Y. Nir, Phys. Lett. B357 (1995) 630.

[154] Y. Kuno, Nucl. Phys. B (Proc. Suppl.) 37A (1994) 87.

[155] Y. Grossman and Z. Ligeti, Phys. Lett. B347 (1995) 399.

[156] Y. Grossman, Nucl. Phys. B426 (1994) 355.

[157] M. Boulware and D. Finnell, Phys. Rev. D44 (1991) 2054.

[158] G.C. Branco and M.N. Rebelo, Phys. Lett. B160 (1985) 117.

[159] J. Liu and L. Wolfenstein, Nucl. Phys. B289 (1987) 1.

[160] J. Liu and L. Wolfenstein, Phys. Lett. B197 (1987) 536.

[161] H. Haber and Y. Nir, Nucl. Phys. B335 (1990) 363.

[162] T.P. Cheng and M. Sher, Phys. Rev. D35 (1987) 3484.

[163] A.S. Joshipura and S.D. Rindani, Phys. Lett. B260 (1991) 149.

[164] A. Antaramian, L.J. Hall and A. Rasin, Phys. Rev. Lett. 69 (1992) 1871.

[165] Y.L. Wu and L. Wolfenstein, Phys. Rev. Lett. 73 (1994) 1762.

[166] G.C. Branco, W. Grimus and L. Lavoura, Phys. Lett. B380 (1996) 119.

[167] D. Atwood, L. Reina and A. Soni, Phys. Rev. D54 (1996) 3269.

[168] M. Leurer, Y. Nir and N. Seiberg, Nucl. Phys. B398 (1993) 319.

[169] N. Arkani-Hamed, C.D. Carone, L.J. Hall and H. Murayama, Phys. Rev. D54 (1996) 7032 .

[170] T.D. Lee, Phys. Rev. D8 (1973) 1226.

[171] C.H. Albright, J. Smith, and S.H.H. Tye, Phys. Rev D21 (1980) 711.

[172] A. Méndez and A. Pomarol, Phys. Lett. B272 (1991) 313.

[173] A. Pomarol and R. Vega, Nucl. Phys. B413 (1993) 3.

[174] C.E. Schmidt and M.E. Peskin, Phys. Rev. Lett. 69 (1992) 410.

[175] D. Chang and W.Y. Keung, Phys. Lett. B305 (1993) 261.

[176] A. Pilaftsis and M. Nowakowski, Int. J. Mod. Phys. A9 (1994) 1097, (E) A9 (1994) 5849.

[177] X.G. He, J.P. Ma and B. McKellar, Mod. Phys. Lett. A9 (1994) 205. 
[178] W. Bernreuther and A. Brandenburg, Phys. Lett. B314 (1993) 104.

[179] H. Anlauf, W. Bernreuther and A. Brandenburg, Phys. Rev. D52 (1995) 3803, (E) D53 (1996) 1725.

[180] B. Grzadkowski, Phys. Lett. B338 (1994) 71.

[181] T. Arens, U.D.J. Gieseler and L.M. Sehgal, Phys. Lett. B339 (1994) 127.

[182] S. Bar-Shalom, D. Atwood, G. Eilam, R.R. Mendel and A. Soni, Phys. Rev. D53 (1996) 1162.

[183] J.F. Gunion and D. Wyler, Phys. Lett. B248 (1990) 170.

[184] E. Braaten, C.S. Li and T.C. Yuan, Phys. Rev. Lett. 64 (1990) 1709.

[185] A. De Rújula, M.B. Gavela, O. Pène and F.J. Vegas, Phys. Lett. B245 (1990) 640.

[186] L. Wolfenstein, Phys. Rev. Lett. 13 (1964) 562.

[187] B. Winstein, Phys. Rev. Lett. 68 (1992) 1271.

[188] J.M. Soares and L. Wolfenstein, Phys. Rev. D46 (1992) 256.

[189] B. Winstein and L. Wolfenstein, Rev. Mod. Phys. 65 (1993) 1113.

[190] J.-M. Gerard and T. Nakada, Phys. Lett. B261 (1991) 474.

[191] L. Lavoura, Int. J. Mod. Phys. A9 (1994) 1873.

[192] D. Chang, Nucl. Phys. B214 (1983) 435.

[193] G.C. Branco, J.-M. Frère and J.-M. Gérard, Nucl. Phys. B221 (1983) 317.

[194] H. Harari and M. Leurer, Nucl. Phys. B233 (1984) 221.

[195] G. Ecker, W. Grimus and H. Neufeld, Nucl. Phys. B247 (1983) 70.

[196] G. Ecker and W. Grimus, Nucl. Phys. B258 (1985) 328.

[197] M. Leurer, Nucl. Phys. B266 (1986) 147.

[198] R.N. Mohapatra and J.C. Pati, Phys. Rev. D11 (1975) 566.

[199] J. Basecq, J. Liu, J. Milutonovic and L. Wolfenstein, Nucl. Phys. B272 (1986) 145.

[200] G. Beall, M. Bander and A. Soni, Phys. Rev. Lett. 48 (1982) 848.

[201] G. Beall and A. Soni, Phys. Rev. Lett. 47 (1981) 552.

[202] X.G. He, H.J. McKellar and S. Pakvasa, Phys. Rev. Lett. 61 (1988) 1267.

[203] D. Chang, C.S. Li and T.C. Yuan, Phys. Rev. D42 (1990) 867.

[204] G. Ecker and W. Grimus, Z. Phys. C30 (1986) 293.

[205] D. London and D. Wyler, Phys. Lett. B232 (1989) 503.

[206] J. Liu, C.Q. Geng and J.N. Ng, Phys. Rev. D39 (1989) 3473. 